\title{
Harmonic functions and instanton moduli spaces on the multi-Taub-NUT space
}

\author{
Gábor Etesi \\ Department of Geometry, Mathematical Institute, Faculty of Science, \\ Budapest University of Technology and Economics, \\ Egry J. u. 1, H ép., H-1111 Budapest, Hungary * \\ Szilárd Szabó \\ Department of Geometry, Mathematical Institute, Faculty of Science, \\ Budapest University of Technology and Economics, \\ Egry J. u. 1, H ép., H-1111 Budapest, Hungary ${ }^{\dagger}$
}

November 5, 2018

\begin{abstract}
Explicit construction of the basic SU(2) anti-instantons over the multi-Taub-NUT geometry via the classical conformal rescaling method is exhibited. These anti-instantons satisfy the so-called weak holonomy condition at infinity with respect to the trivial flat connection and decay rapidly. The resulting unit energy anti-instantons have trivial holonomy at infinity.

We also fully describe their unframed moduli space and find that it is a five dimensional space admitting a singular disk-fibration over $\mathbb{R}^{3}$.

On the way, we work out in detail the twistor space of the multi-Taub-NUT geometry together with its real structure and transform our anti-instantons into holomorphic vector bundles over the twistor space. In this picture we are able to demonstrate that our construction is complete in the sense that we have constructed a full connected component of the moduli space of solutions of the above type.

We also prove that anti-instantons with arbitrary high integer energy exist on the multiTaub-NUT space.
\end{abstract}

AMS Classification: Primary: 53C07; Secondary: 14F05, 53C28, 58J99

Keywords: Multi-Taub-NUT space; Yang-Mills instantons; Harmonic functions; Twistors

*e-mail: etesi@math.bme.hu

$\dagger$ e-mail: szabosz@math. bme.hu 


\section{Introduction}

The aim of this paper is to construct the most relevant anti-instanton moduli space over the multi-Taub-NUT geometry by elementary means.

An important class of non-compact but complete four dimensional geometries is the collection of the so-called asymptotically locally flat (ALF) spaces including several mathematically as well as physically important examples. The flat $\mathbb{R}^{3} \times S^{1}$ plays a role in finite temperature Yang-Mills theories, the Euclidean Schwarzschild space [20] deals with quantum gravity and Hawking radiation. If the metric is additionally hyper-Kähler then the space is also called an ALF gravitational instanton (in the narrow sense). The flat $\mathbb{R}^{3} \times S^{1}$ is a straightforward example; non-trivial ones for this restricted class are provided by the multi-Taub-NUT (or $A_{k}$ ALF or ALF Gibbons-Hawking) spaces [15] which carry supersymmetric solutions of string theory and supergravity models, the Atiyah-Hitchin manifold (and its universal double cover) [3] describing the 2-monopole moduli space over $\mathbb{R}^{3}$ and last but not least the recently constructed $D_{k}$ ALF spaces [9].

The ALF asymptotics is a natural generalization of the well-known ALE (asymptotically locally Euclidean) one including the multi-Eguchi-Hanson geometries [15]. Instanton theory over these later spaces possessing several phenomena related with non-compactness (e.g. existence of four dimensional moduli spaces isometric to the original space) is well-known due to the important paper of Kronheimer and Nakajima [27] in which a full ADHM construction was established. The existence of this construction is in some sense not surprising because the original ADHM construction was designed for the flat $\mathbb{R}^{4}$ and all ALE spaces arise by an algebro-geometric deformation of the flat quotients $\mathbb{C}^{2} / \Gamma$ where $\Gamma \subset \mathrm{SU}(2)$ are various discrete subgroups [26].

The natural question arises: what about instanton theory over ALF spaces? Unlike the ALE geometries, these are essentially non-flat spaces in the sense above therefore one may expect that instanton theory somewhat deviates from that over the flat $\mathbb{R}^{4}$. Some general questions have been answered recently [13] and these investigations pointed out that in spite of their more transcendental nature, open Riemannian spaces with ALF asymptotics rather resemble compact four manifolds at least from the point of view of instanton theory. To test this interesting observation more carefully in this paper we work out the simplest moduli space over the multiTaub-NUT geometry: we will find that this moduli space is five dimensional furthermore its part containing concentrated instantons looks like a "collar" of the original manifold, supporting the analogy with the compact case. We note that this apparent compactness is related to the existence of a smooth compactification of ALF spaces motivated by $L^{2}$ cohomology theory [19].

The paper is organized as follows. In Sect. 2 we quickly summarize two important tools for solving the self-duality equations over an anti-half-conformally flat space: the conformal rescaling method of Jackiw-Nohl-Rebbi [25, 4] as well as the Atiyah-Ward correspondence [5]. Via conformal rescaling one constructs instantons out of positive harmonic functions with at most pointlike singularities and appropriate decay toward infinity while the Atiyah-Ward correspondence establishes a link between instantons and holomorphic vector bundles. Since these methods meet in Penrose' twistor theory we shall also briefly outline it here.

Then we introduce ALF spaces. Referring to recent results on instanton theory over these spaces [13] we precisely define the class of anti-instantons which are expected to form nice moduli spaces. These solutions must obey two conditions: the so-called weak holonomy condition with respect to some smooth flat connection and the rapid decay condition (cf. Definition 2.1 here). The former condition, albeit looks like an analytical one, in fact deals with the topology of infinity of the ALF space only [13, Theorem 2.3] meanwhile the latter one controls the fall-off properties 
of an anti-instanton hence is indeed analytical in its nature.

In Sect. 3, taking probably the most relevant ALF example namely the multi-Taub-NUT series, following Andreson-Kronheimer-LeBrun [1] and LeBrun [28] we review this geometry with special attention to the description of its generic complex structures as algebraic surfaces in $\mathbb{C}^{3}$. We also identify a finite number of distinguished complex structures that can be realized as blowups of these algebraic surfaces. The group $S^{1}$ acts on these spaces via isometries and this action has isolated fixed points, called NUTs.

Then in Sect. 4 we come to our main results. We shall prove via conformal rescaling that over the multi-Taub-NUT space $\left(M_{V}, g_{V}\right)$ unframed $L^{2}$ moduli spaces of SU(2) anti-instantons obeying both the aforementioned weak holonomy condition with respect to the trivial flat connection $\nabla_{\Theta}$ as well as the rapid decay condition are not empty. Since the input in this construction is a positive harmonic function, the so-called non-parabolic manifolds [17, 21, 34] are the key concept here which admit an abundance of positive minimal Green functions $G(\cdot, y)$ with arbitrarily prescribed pointlike singularity $y \in M_{V}$ (cf. Definition 4.1 here). Non-parabolicity deals with the volume growth toward infinity and is closely related to the ALF property of our space as it was observed by Minerbe [29, Theorem 0.1]. These functions provide us with a five real paremeter family

$$
f_{y, \lambda}=1+\lambda G(\cdot, y)
$$

of positive harmonic functions hence that of non-gauge equivalent irreducible SU(2)-anti-selfdual connections of unit energy on the positive chiral spinor bundle - parameterized by a point $y \in M_{V}$ and a "concentration parameter" $\lambda \in(0,+\infty)$. These solutions obey the weak holonomy condition with respect to the trivial flat connection as well as decay rapidly. This way we obtain a familiar "collar" for the original manifold (cf. Theorem 4.1 here). Since we know from [13, Theorem 3.2] that the unit energy unframed moduli space of these irreducible solutions is a smooth five dimensional manifold it follows that this collar in fact realizes an open subset of it (or one of its connected component) and describes the regime of concentrated unit energy anti-instantons with trivial holonomy at infinity.

Then we proceed further and construct additional anti-instantons in the "centerless" direction i.e., when the concentration parameter $\lambda$ becomes infinite. These solutions just correspond to pure Green functions. However in this limit anti-instantons corresponding to the NUTs are reducible to $\mathrm{U}(1)$ consequently it is natural to talk about (a connected component of) the unframed moduli space $\widehat{\mathscr{M}}(1, \Theta)$ including these reducible solutions as well. Therefore we will find in particular that this extended moduli space is not a manifold anymore rather admits usual singularities in the reducible points moreover that our moduli space itself admits a singular fibration

$$
\Phi: \widehat{\mathscr{M}}(1, \Theta) \longrightarrow \mathbb{R}^{3}
$$

whose generic fibers are 2 dimensional open while the singular ones are 1 dimensional semi-open balls (cf. Theorem 4.2 here). The aforementioned anti-instantons with infinite concentration parameter $\lambda=+\infty$ are represented in the generic case by the centers of these open 2-balls meanwhile in the singular case by the closed end of these semi-open 1-balls. For a given generic fiber the $\lambda=$ const. $<+\infty$ circles around the center describe anti-instantons "centered" about points in $\left(M_{V}, g_{V}\right)$ along the same isometry orbit and with fixed finite concentration parameter. The singular fibers correspond to the fixed points of the isometric $S^{1}$-action hence in a given singular fiber there is a unique anti-instanton with $\lambda=$ const. $\leq+\infty$. In other words, all "centerless" anti-instantons stemming from pure Green functions with pointlike singularities along a given isometry orbit are gauge equivalent. 
We will also be able to demonstrate that similar moduli spaces with arbitrary high energy and trivial holonomy at infinity are non-empty according to previous expectations.

For a comparison we also construct the moduli space of rapidly decaying unit energy $\mathrm{SU}(2)$ instantons with trivial holonomy in infinity over the flat $\mathbb{R}^{3} \times S^{1}$ (cf. Theorem 4.4 here).

In the last section, Sect. 5 we round things off by proving, as already mentioned, that our construction is complete in the sense that at least a whole connected component of the basic moduli space emerges this way. This will require however a quite tedious work.

To this end first we provide a detailed construction of the twistor space of the multi-TaubNUT geometry together with its real structure $\grave{a}$ la Hitchin $[6,22,24]$. Then we transform our anti-instantons constructed out of harmonic functions into holomorphic vector bundles on the twistor space in the spirit of the Atiyah-Ward correspondence [5]. More precisely, by the aid of Atiyah [2] we work out a smooth compactification of the twistor space which makes it possible to identify the harmonic functions used to construct anti-instantons with elements of certain Ext groups of the compactified twistor space (cf. Theorem 5.1 here). A novelty of our compactification compared to Atiyah's is that our resulting Ext groups have the correct dimension (cf. Lemma 5.5) i.e., they inculde the constant harmonic functions as well. (Note that without compactification the Ext groups would be infinite dimensional.)

These Ext groups can be used to obtain holomorphic bundles on the twistor space. In particular gluing data of the bundles corresponding to minimal Green functions can be worked out explicitly [2] and one can conclude that the isometric $S^{1}$-action induces an isomorphism of these bundles therefore the associated "centerless" anti-instantons indeed must be gauge equivalent. This provides us with the picture of the moduli space mentioned above.

Working with the twistor space also can be viewed as a step toward establishing a general ADHM-like instanton factory [8] which can be used to replace the conformal rescaling method; this is certainly necessary since our technique is not capable to grasp all higher energy moduli spaces.

We conjecture that in fact we have obtained the full moduli space of unit energy, rapidly decaying anti-instantons with trivial holonomy at infinity in this paper i.e., the basic moduli space is connected.

Finally for a comparison we make a comment here on other gravitational instantons of ALG and ALH type introduced by Cherkis and Kapustin. By their definition, these geometries are parabolic hence one cannot expect the existence of positive minimal Green functions over them and accordingly, a canonical five parameter family of anti-instantons. Indeed, the simplest ALG space is the flat $\mathbb{R}^{2} \times T^{2}$ and it is known that the framed unit energy anti-instanton moduli space here is one dimensional only [7]. Hence in the ALE-ALH hierarchy apparently ALF spaces represent the closest analogues of compact four-manifolds from an instanton theoretic viewpoint. Analogously, in this hierarchy ALF spaces are the only ones whose $L^{2}$ cohomological compactification [19] is smooth.

Acknowledgement. The second author would like to thank the Alfréd Rényi Institute of Mathematics, where he had a scholarship during the early stages of this article, and Vincent Minerbe for useful discussions. Both authors were partially supported by OTKA grant No. NK81203 (Hungary). 


\section{Constructing instantons}

In this section we quickly summarize two methods to obtain instantons over an anti-self-dual Riemannian manifold: the conformal rescaling method and the Atiyah-Ward correspondence. These two approaches meet each other in twistor theory therefore we also review the basic facts about it here.

Let us begin with the conformal rescaling method which was first used by Jackiw, Nohl and Rebbi to obtain instantons over the flat $\mathbb{R}^{4}$ [25]. Remember that an $\mathrm{SU}(2)$ (anti-)self-dual connection $\nabla_{A}$ over a four dimensional oriented Riemannian manifold $(M, g)$ is a smooth $\mathrm{SU}(2)$ connection on any $\mathrm{SU}(2)$ rank two complex vector bundle $E$ over $M$ whose curvature satisfies the (anti-)self-duality equations $*_{g} F_{A}= \pm F_{A}$. The gauge equivalence class $\left[\nabla_{A}\right]$ of this connection is called an (anti-)instanton if additionally its energy is finite: $\left\|F_{A}\right\|_{L^{2}(M)}<+\infty$. Note that this assumption on the energy is non-trivial only if $(M, g)$ is non-compact but complete.

Let $(M, g)$ be an oriented Riemannian spin four-manifold, possibly non-compact but complete. Take the Levi-Civita connection $\nabla$ of the metric and lift it to get the corresponding spin connection $\nabla^{s}$. Referring to the splitting $\mathfrak{s o}(4)=\mathfrak{s u}(2)^{+} \oplus \mathfrak{s u}(2)^{-}$the projected connection $\nabla_{A}:=p^{+}\left(\nabla^{s}\right)$, with $p^{+}: \mathfrak{s o}(4) \rightarrow \mathfrak{s u}(2)^{+}$being the canonical projection, is an $\mathrm{SU}(2)^{+}$connection on the $\mathrm{SU}(2)^{+}$rank two complex vector bundle $\Sigma^{+}$(the positive chiral spinor bundle).

Assume $(M, g)$ is moreover anti-half-conformally flat i.e., $W_{g}^{+}=0$. Picking up an everywhere smooth and positive function $f$ on $M$, one can consider the conformally rescaled metric $\tilde{g}:=f^{2} g$. The new space $(M, \tilde{g})$ still satisfies $W_{\tilde{g}}^{+}=0$ by conformal invariance of the Weyl-tensor. Its scalar curvature however transforms as $f^{3} s_{\tilde{g}}=6 \triangle_{g} f+f s_{g}$ where $\triangle_{g}$ refers to the scalar Laplacian on $(M, g)$. If this new scalar curvature $s_{\tilde{g}}$ happens to vanish over $M$ i.e., the rescaling function satisfies the equation

$$
\triangle_{g} f+\frac{s_{g}}{6} f=0
$$

then the corresponding projected connection $\nabla_{\tilde{A}}$, constructed like $\nabla_{A}$ before, satisfies the antiself-duality equations $*_{\tilde{g}} F_{\tilde{A}}=-F_{\tilde{A}}$ over $(M, \tilde{g})$ by a variant of the Atiyah-Hitchin-Singer theorem [4, Proposition 2.2]. However taking into account the conformal invariance of self-duality, we obtain that $\nabla_{\tilde{A}}$ in fact satisfies the original $\mathrm{SU}(2)^{+}$anti-self-duality equations $*_{g} F_{\tilde{A}}=-F_{\tilde{A}}$ over $(M, g)$. Hence we have construcred a solution of the anti-self-duality equation via the conformal rescaling method.

This anti-self-dual connection locally can be constructed as follows. Let $U \subset M$ be a coordinate ball and let $\left(\xi^{0}, \ldots, \xi^{3}\right)$ denote the orthonormal frame field which diagonalizes $\left.g\right|_{U}$. In this gauge write $\left.\nabla\right|_{U}=\mathrm{d}+\omega$ for the Levi-Civita connection. The rescaled metric $f^{2} g$ has a corresponding local frame field $\left(f \xi^{0}, \ldots, f \xi^{3}\right)$ and Levi-Civita connection $\left.\widetilde{\nabla}\right|_{U}=\mathrm{d}+\tilde{\omega}$. The connection 1-form $\tilde{\omega}$ can be calculated by the aid of the Cartan equation $\mathrm{d}\left(f \xi^{i}\right)+\tilde{\omega}_{j}^{i} \wedge\left(f \xi^{j}\right)=0$. An easy computation shows that

$$
\tilde{\omega}_{j}^{i}=\omega_{j}^{i}+\left((\mathrm{d} \log f) \xi_{i}\right) \xi^{j}-\left((\mathrm{d} \log f) \xi_{j}\right) \xi^{i}
$$

where $\left(\xi_{0}, \ldots, \xi_{3}\right)$ denotes the dual vector field on $T U$ (with respect to the original metric $\left.g\right|_{U}$ ) and $(\mathrm{d} \log f) \xi_{i}=\left.g\right|_{U}\left(\operatorname{grad}(\log f), \xi_{i}\right)$. Lifting $\widetilde{\nabla}$ to the spin bundle then projecting it to the positive side (using the 't Hooft matrices, cf. [12]) and exploiting the identification $\mathfrak{s u}(2)^{+} \cong \operatorname{Im} \mathbb{H}$ given by $\left(\sigma_{1}, \sigma_{2}, \sigma_{3}\right) \mapsto(\mathbf{i},-\mathbf{j},-\mathbf{k})$ we obtain in this induced gauge

$$
\tilde{A}=A+\frac{1}{2} \operatorname{Im}((\mathbf{d} \log f) \boldsymbol{\xi})
$$


with $A:=p^{+}\left(\omega^{s}\right)$ being the projection of the original spin connection $\left.\nabla^{s}\right|_{U}=\mathrm{d}+\omega^{s}$ and the quaternion-valued 0 -form $\mathbf{d} \log f$ and 1 -form $\boldsymbol{\xi}$ in the second term are defined respectively as follows:

$$
\mathbf{d} \log f:=\mathrm{d} \log f\left(-\xi_{0}+\xi_{1} \mathbf{i}+\xi_{2} \mathbf{j}+\xi_{3} \mathbf{k}\right), \quad \boldsymbol{\xi}:=\xi^{0}+\xi^{1} \mathbf{i}+\xi^{2} \mathbf{j}+\xi^{3} \mathbf{k} .
$$

Writing $\left.a\right|_{U}:=\frac{1}{2} \operatorname{Im}((\mathbf{d} \log f) \boldsymbol{\xi})$ for the only term involving $f$, it is clear that it extends over $M$ as an $\operatorname{Im} \mathbb{H}$-valued 1 -form i.e., one finds

$$
a \in C^{\infty}\left(M ; \Lambda^{1} M \otimes \operatorname{End} \Sigma^{+}\right)
$$

and it is insensitive for an overall rescaling of $f$ by a non-zero real constant. This implies that from the point of view of our anti-instanton factory, we can regard two conformal scaling functions identical if they differ by an overall rescaling only. Moreover note that the new connection takes the form $\nabla_{\tilde{A}}=\nabla_{A+a}$ i.e., this whole conformal rescaling procedure can be regarded as an antiself-dual perturbation of the original connection. For an explicit application of this method we refer to [25] over the flat $\mathbb{R}^{4}$ and to [12] in case of the multi-Taub-NUT space.

If $(M, g)$ is compact then this machinery provides us with an anti-instanton on $\Sigma^{+}$. If the space is non-compact but complete and $\nabla_{A}$ has finite energy (which can be achieved if the metric decays sufficiently fast to the flat one at infinity as we have seen) then if the perturbation $a$ also decays fast i.e., $f$ is sufficiently bounded at infinity then $\nabla_{\tilde{A}}$ has still finite energy consequently we obtain an anti-instanton on the same bundle $\Sigma^{+}$over $(M, g)$.

An important particular case is when the original space $(M, g)$ has already vanishing scalar curvature. In this case (1) cuts down to the ordinary Laplace equation $\triangle_{g} f=0$ and the original connection $\nabla_{A}$ is already anti-self-dual corresponding to the trivial solution $f=1$.

However it is clear by the maximum principle that in many situations there are no nontrivial everywhere smooth bounded solutions to (1) therefore this method is apparently vacuous. Fortunately we can modify $f$ to possess mild singularities over $M$. At this level of generality by a "mild singularity" we mean the following:

(i) There are finitely many points $\left\{y_{1}, \ldots, y_{k}\right\} \subset M$ such that $f$ diverges in $y_{i}$ for all $i=$ $1,2, \ldots k$

(ii) The local energy of the rescaled connection around each point is finite i.e., for $U_{i}$ a neighbourhood of $y_{i}$ one has

$$
\left\|F_{\tilde{A}}\right\|_{L^{2}\left(U_{i} \backslash\left\{y_{i}\right\}\right)}<+\infty
$$

for all $i=1,2, \ldots, k$. (In terms of $f$ this condition simply controls its behaviour about $y_{i}$.)

Under these circumstances Uhlenbeck's theorem [33] allows us to remove the singularities from $\nabla_{\tilde{A}}$ introduced by $f$. Therefore if $f$ is a positive solution to (1) with such singularities then the resulting anti-self-dual connection will be smooth again and corresponds to a non-trivial antiinstanton over a bundle $E$ if $M$ is compact. This $\mathrm{SU}(2)^{+}$bundle may be no more isomorphic to $\Sigma^{+}$as a consequence of the allowed singularities of the scaling function. In fact its topological type is determined by the singularity structure of $f$. In case of a non-compact complete base geometry if in addition $f$ is sufficiently bounded at infinity then the resulting anti-self-dual connection has finite energy i.e., describes an anti-instanton on the same bundle $\Sigma^{+}$, regardless how many singularities $f$ possesses. This is because in fact over a non-compact four-manifold all $\mathrm{SU}(2)^{+}$bundles, including $\Sigma^{+}$, are isomorphic to the trivial one. The significance of the singularity structure of scaling functions in instanton theory therefore becomes transparent. 
We move on and recall twistor theory of anti-half-conformally flat geometries and in particular the Atiyah-Ward construction [5] which is another powerful way to solve the (anti-)self-duality equations at least in principle.

Let us recall the general theory [6, Chapter 13]. Let $(M, g)$ be an oriented Riemannian fourmanifold and consider the projectivization $Z:=P\left(\Sigma^{+}\right)$of the positive chiral spinor bundle $\Sigma^{+}$ on $M$ (this projectivization is well-defined even if $M$ is not spin). Clearly, $Z$ admits a fiber bundle structure $p: Z \rightarrow M$ with $\mathbb{C}^{1}$ 's as fibers. A fiber $F_{x}$ over $x \in M$ can be viewed as the parameter space of orthogonal complex structures on $T_{x} M$ whose induced orientations agree with that of $M$. Using the Levi-Civita connection on $(M, g)$ we can split the tangent space $T_{z} Z$ at any point $z \in Z$; writing $z=(x, p)$ with $x \in M$ and $p \in F_{x}$ one endows the horizontal subspace $H_{(x, p)} \cong T_{x} M$ of $T_{(x, p)} Z$ with the complex structure $J_{p}$ given by $p \in F_{x}$ itself meanwhile the vertical subspace $V_{(x, p)} \cong T_{p} F_{x}$ of $T_{(x, p)} Z$ is equipped with the standard complex structure $I$ coming from $F_{x} \cong \mathbb{C} P^{1}$. Therefore $T_{(x, p)} Z$ carries a complex structure $\left(J_{p}, I\right)$ and $Z$ as a real six-manifold gets possess an almost complex structure. A basic theorem of Penrose [32] or Atiyah, Hitchin and Singer [4, Theorem 4.1][6, Theorem 13.46] states that this complex structure is integrable i.e., $Z$ is a complex three-manifold if and only if $(M, g)$ is anti-half-conformally flat. The space $Z$ is called the twistor space of $(M, g)$. The complex structure in fact depends on the confomal class of the metric only.

The basic holomorphic structure of $Z$ looks like as follows [6, Chapter 13]:

(i) For all $y \in M$ the fibers $F_{y} \subset Z$ represent holomorphic lines $Y \subset Z$ with normal bundles isomorphic to $H \oplus H$ where $H$ is the usual line bundle $H$ with $\left\langle c_{1}(H),[Y]\right\rangle=1$ on $Y \cong \mathbb{C} P^{1}$;

(ii) The antipodal map on $Y$ induces a real structure on $Z$ i.e., an anti-holomorphic involution $\tau: Z \rightarrow Z$ satisfying $\tau^{2}=\operatorname{Id}_{Z}$;

(iii) The space of all holomorphic lines in $Z$ near the fibers $Y$ form a locally complete family which is a complex manifold $M^{\mathbb{C}}$ of complex dimension four and the fibers $Y$ among them are dinstinguished by the property that the real structure fixes them, hence are called real lines.

A remarkable property of twistor spaces is that they allow one to solve certain linear or non-linear field equations over $(M, g)$. These equations are of great importance in physics. We shall also quickly review these constructions.

1. The linear field equation relevant to us here is the conformal scalar Laplace equation (1). We shall denote by $H_{Z}$ the holomorphic line bundle on the whole $Z$ such that for any fiber the restriction $\left.H_{Z}\right|_{Y}$ is the standard line bundle $H$ on $Y \cong \mathbb{C} P^{1}$ and by $H_{Z}^{k}$ its $k$ th tensor product. For notational convenience write $\mathscr{O}_{Z}(k)$ for the sheaf $\mathscr{O}\left(H_{Z}^{k}\right)$. Recall (cf. e.g. [23]) that complexvalued real analytic functions satisfying (1) on open subsets $\left(U,\left.g\right|_{U}\right) \subset(M, g)$ are in one-to-one correspondence with elements of the sheaf cohomology group $H^{1}\left(p^{-1}(U) ; \mathscr{O}_{p^{-1}(U)}(-2)\right)$ i.e., there is a natural isomorphism

$$
T:\left.H^{1}\left(p^{-1}(U) ; \mathscr{O}_{p^{-1}(U)}(-2)\right) \cong \operatorname{Ker} \triangle_{g}\right|_{U}
$$

called the Penrose transform: given an element $\varphi \in H^{1}\left(p^{-1}(U) ; \mathscr{O}_{p^{-1}(U)}(-2)\right)$ and a real line $Y \subset p^{-1}(U)$ one can take the restriction $\left.\varphi\right|_{Y} \in H^{1}\left(Y ; \mathscr{O}_{Y}(-2)\right) \cong \mathbb{C}$. This gives rise to a complex-valued function $f(y):=\left.\varphi\right|_{Y}$ consequently one has a map

$$
T: H^{1}\left(p^{-1}(U) ; \mathscr{O}_{p^{-1}(U)}(-2)\right) \longrightarrow C^{\infty}(U, \mathbb{C}) .
$$


It turns out that $f$ is a solution to (1) on $U \subset M$ and all local solutions arise this way.

2. The non-linear (anti-)self-duality equations of Yang-Mills theory, also can be treated in twistor theory (cf. e.g. [4]): solutions of the $\mathrm{SU}(2)$ anti-self-duality equations over $(M, g)$ with approriate orientation can be converted into certain holomorphic vector bundles on $Z$ as follows. There is a one-to-one correspondence of gauge equivalence classes of anti-self-dual SU(2) connections on a rank two complex vector bundle $E$ on $M$ and isomorphism classes of rank two complex vector bundles $F$ on $Z$ such that

(i) $F$ is holomorphic;

(ii) For any real line $Y \subset Z$ the restricted bundle $\left.F\right|_{Y}$ is holomorphically trivial;

(iii) There exists a conjugate linear map $\tilde{\tau}: F \rightarrow F$ lying over the real structure $\tau: Z \rightarrow Z$ and satisfies $\tilde{\tau}^{2}=-\operatorname{Id}_{F}$.

If $\nabla_{A}$ is irreducible and $Z$ (that is, $M$ ) is compact then $F$ is a stable holomorphic bundle on $Z$ and in this case $\tilde{\tau}$ is unique up to scalar multiplication.

This construction, called the Atiyah-Ward correspondence, goes as follows. Take an SU(2) vector bundle $E$ and an anti-self-dual connection on it over $(M, g)$. Let $F:=p^{*} E$ be a $C^{\infty}$ bundle; by anti-self-duality the curvature of the lifted connection $p^{*} \nabla_{A}$ will be of $(1,1)$-type hence $F$ will be holomorphic. One checks that properties (ii) and (iii) are also satisfied. Conversely, take a holomorphic bundle $F$ satisfying (ii) and (iii). Define $E_{y}$ to be the space of holomorphic sections over $\left.F\right|_{Y}$ (this space is two complex dimensional via (ii)). Property (iii) induces a symplectic structure on $F$ consequently we can take the corresponding unique unitary connection $\nabla$ on $F$. By uniquenss and (ii) this connection is the trivial connection on each $\left.F\right|_{Y}$ hence is of the form $p^{*} \nabla_{A}$ with a connection $\nabla_{A}$ on $E$. Since the curvature of this connection is of $(1,1)$-type with respect to all complex structures on $T_{y} M$ for all $y \in M$ it follows that it is anti-self-dual.

3. Another non-linear equation, namely the (Riemannian) anti-self-dual vacuum Einstein equations of general relativity can also be naturally adjusted to twistor theory, known as Penrose' non-linear graviton construction $[32,22]$. Assume here that $(M, g)$ is moreover simply connected and Ricci-flat i.e., simply connected and anti-self-dual. Then it follows that the induced connection on $\Sigma^{+}$is the trivial flat one and $Z$ can be retracted onto its particular fiber $F_{y}$ by parallel transport. Therefore topologically $Z \cong M \times S^{2}$ in this case. The associated twistor space of $(M, g)$ has in addition to the basic holomorphic properties above the following:

(i) There is a holomorphic fibration $\pi: Z \rightarrow Y$ over a particular line $Y \cong \mathbb{C} P^{1}$ with fibers being the original space $M$. Consequently $H_{Z}^{k} \cong \pi^{*} H^{k}$ for all $k \in \mathbb{Z}$;

(ii) Since the complex normal bundle $N_{Y}$ of each $Y$ is isomorphic to $H \oplus H$ it follows that the canonical bundle $K_{Z}$ of $Z$ is isomorphic to $\pi^{*} H^{-4}$.

Conversely, it can be shown [6, Chapter 13] that a complex 3 -space $Z$ having these properties encodes the conformal class of an anti-self-dual simply connected four-space $(M, g)$. Indeed, the complexified space $M^{\mathbb{C}}$ carries a natural conformal structure by declaring two points $Y^{\prime}, Y^{\prime \prime} \in M^{\mathbb{C}}$ to be null-separated if and only if for the corresponding lines $Y^{\prime} \cap Y^{\prime \prime} \neq \emptyset$ in $Z$. Restricting this conformal structure to the real lines parameterized by $M \subset M^{\mathbb{C}}$ one comes up with the confomal class of an anti-self-dual structure $(M, g)$. The particular metric emerges via an appropriate isomorphism $K_{Z} \cong \pi^{*} H^{-4}$. 
We have encountered two techniques for constructing anti-instantons over an anti-half-conformally flat space. The link between them is an observation of Atiyah [2] which relates solutions to (1) to holomorphic vector bundles on the twistor space. We will work out this in detail in Sect. 5.

In the rest of this section we introduce a special class of four-manifolds: suppose from now on that $(M, g)$ is a four dimensional asymptotically locally flat (ALF) space as it was defined in [13]. By a recent powerful theorem of Minerbe [29, Theorem 0.1] these four dimensional geometries at least in the connected, geodesically complete, hyper-Kähler case can also be characterized by requiring their curvature to be $L^{2}$ with respect to the measure $\frac{r^{4}}{\operatorname{Vol}_{g}\left(B_{r}^{4}(x)\right)} \mathrm{d} V_{g}$ as well as prescribing their asymptotical volume growth to be $O\left(r^{\nu}\right)$ with $3 \leq \nu<4$.

Framed moduli spaces of certain "admissible" $\mathrm{SU}(2)$ (anti-)instantons over these geometries have been investigated recently [13]. By "admissible" we mean the following. Topologically, an $\operatorname{ALF}$ space $(M, g)$ (with a single end) admits a decomposition $M=K \cup W$ where $K$ is a compact interior space and $W$ is an end or neck homeomorphic to $N \times \mathbb{R}^{+}$where $\pi_{N}: N \rightarrow B_{\infty}$ is a connected, compact, oriented three-manifold fibered over a compact Riemann surface $B_{\infty}$ with circle fibers $F \cong S^{1}$. Note that for any $x \in M$ and sufficiently large $R>0 \mathrm{~W}$ is homeomorphic to $M \backslash B_{R}^{4}(x)$ where $B_{R}^{4}(x)$ is a geodesic ball about $x \in M$ with radius $R>0$.

Definition 2.1. Let $(M, g)$ be an ALF four-manifold. Take an arbitrary smooth, finite energy $\mathrm{SU}(2)$ connection $\nabla_{A}$ on a (necessarily trivial) $\mathrm{SU}(2)$ vector bundle $E$ over $M$. This connection is said to be admissible if it satisfies two conditions (cf. [13]):

(i) The first is called the weak holonomy condition and says that to $\nabla_{A}$ there exists a smooth flat $\mathrm{SU}(2)$ connection $\left.\nabla_{\Gamma}\right|_{W}$ on $\left.E\right|_{W}$ along the end $W \subset M$ and a constant $c_{1}=c_{1}(g)>0$, independent of $R>0$, such that in any smooth gauge on $M \backslash B_{R}^{4}(x)$ the inequality

$$
\|A-\Gamma\|_{L_{1, \Gamma}^{2}\left(M \backslash B_{R}^{4}(x)\right)} \leq c_{1}\left\|F_{A}\right\|_{L^{2}\left(M \backslash B_{R}^{4}(x)\right)}
$$

holds along the neck $M \backslash B_{R}^{4}(x)$;

(ii) The second condition requires $\nabla_{A}$ to decay rapidly at infinity i.e.,

$$
\lim _{R \rightarrow \infty} \sqrt{R}\left\|F_{A}\right\|_{L^{2}\left(M \backslash B_{R}^{4}(x)\right)}=0 .
$$

The first condition ensures us that the connection has a well-defined holonomy at infinity given by $\left.\nabla_{\Gamma}\right|_{W}$; however note that if the infinity is not simply connected then this holonomy can in principle be non-trivial. ${ }^{1}$ The second condition regulates how fast the finite energy connection decays to this flat connection and also can be reformulated (cf. [13]) by saying that $F_{A}$ belongs to a weighted Sobolev space with weight $\delta=\frac{1}{2}$ i.e., $F_{A} \in L_{\frac{1}{2}}^{2}\left(M ; \Lambda^{2} M \otimes\right.$ End $\left.E\right)$. Note that this second condition is stronger than the finite energy requirement on $\nabla_{A}$. It was demonstrated in [13] that irreducible $\mathrm{SU}(2)$ anti-instantons satisfying conditions (i) and (ii) with a fixed limiting flat connection $\left.\nabla_{\Gamma}\right|_{W}$ and energy $e$ form framed moduli spaces $\mathscr{M}(e, \Gamma)$ which are smooth finite dimensional manifolds. Their dimensions have also been calculated by the aid of a dimension formula.

Now take an $\operatorname{ALF}$ space $(M, g)$ with a fixed orientation whose curvature satisfies $W_{g}^{+}=0$ and $s_{g}=0$ therefore the Atiyah-Hitchin-Singer theorem applies and the projected Levi-Civita

\footnotetext{
${ }^{1}$ In this paper the case of the trivial holonomy will be considered only.
} 
connection $\nabla_{A}$ is an anti-self-dual connection on $\Sigma^{+}$. By an appropriate curvature decay imposed on the metric (which is incorporated into the precise definition of an ALF space, cf. [13]) this connection has not only finite energy but also decays rapidly as in (ii) of Definition 2.1; moreover if additionally it satisfies the weak holonomy condition (i) in Definition 2.1 then it gives rise to an anti-instanton on $\Sigma^{+}$in the sense above. Performing a conformal rescaling of the metric we obtain another anti-self-dual connection $\nabla_{\tilde{A}}$ on $\Sigma^{+}$. Since it is of the form $\nabla_{A+a}$ with $a$ as in (3) except finitely many points, its curvature reads as $F_{\tilde{A}}=F_{A+a}=F_{A}+\mathrm{d}_{A} a+a \wedge a$ consequently for its energy

$$
\left\|F_{\tilde{A}}\right\|_{L_{\frac{1}{2}}^{2}(M)} \leq\left\|F_{A}\right\|_{L_{\frac{1}{2}}^{2}(M)}+\left\|\mathrm{d}_{A} a\right\|_{L_{\frac{1}{2}}^{2}(M)}+c_{2}\|a\|_{L_{\frac{1}{2}, 1, \Gamma}^{2}(M)}^{2}
$$

holds with a constant $c_{2}=c_{2}(g)>0$. We conclude that the regularity and decay properties of the perturbed connection is determined by the perturbation term. More precisely, the conditions

$$
a \in L_{\frac{1}{2}, 1, \Gamma}^{2}\left(M ; \Lambda^{1} M \otimes \operatorname{End} \Sigma^{+}\right), \tilde{A} \in L_{l o c, 1, \tilde{A}}^{2}\left(M ; \Lambda^{1} M \otimes \operatorname{End} \Sigma^{+}\right), F_{\tilde{A}} \in L_{l o c}^{2}\left(M ; \Lambda^{-} M \otimes \operatorname{End} \Sigma^{+}\right)
$$

ensure us that $a$ affects neither the asymptotics of $\nabla_{A}$ or its fall-off and any pointlike singularities are removable. Therefore if $\nabla_{A}$ is smooth and obeys both the weak holonomy and the rapid decay conditions above then $\nabla_{\tilde{A}}=\nabla_{A+a}$ will be also smooth, decays rapidly and converges to the same limiting flat connection. More precisely one can show that the energy $\tilde{e}$ of $\nabla_{\tilde{A}}$ can differ from that of $\nabla_{A}$ by a non-negative integer only [13]. That is, $\left[\nabla_{A}\right] \in \mathscr{M}(e, \Gamma)$ implies $\left[\nabla_{\tilde{A}}\right] \in \mathscr{M}(\tilde{e}, \Gamma)$ and $\tilde{e}-e \in\{0,1,2, \ldots\}$. In fact as one expects, the difference $\tilde{e}-e$ is governed by the singularity structure of the perturbation i.e., of the scaling function $f$ in (1).

In the next sections we will apply the conformal rescaling technique and twistor theory to understand the moduli space over the multi-Taub-NUT geometries of unit energy SU(2) antiinstantons decaying rapidly and obeying the weak holonomy condition with respect to the trivial connection $\nabla_{\Theta}$.

\section{Review of the multi-Taub-NUT geometry}

In this section we take a closer look of the multi-Taub-NUT spaces following $[1,28]$. Consider $s>0$ distinct points $q_{1}, \ldots, q_{s} \in \mathbb{R}^{3}$. We will construct a four-manifold $M_{V}$ and a smooth map $\pi: M_{V} \rightarrow \mathbb{R}^{3}$ such that $\pi^{-1}\left(q_{j}\right)$ is a point for all $j=1,2, \ldots, s$ but $\pi^{-1}(x) \cong S^{1}$ for all other points $x \in \mathbb{R}^{3} \backslash\left\{q_{1}, \ldots, q_{s}\right\}$. To begin with, let $\pi: U_{V} \rightarrow \mathbb{R}^{3} \backslash\left\{q_{1}, \ldots, q_{s}\right\}$ be the principal $S^{1}$ bundle whose Chern class is -1 when restricted to a small sphere of radius $r_{j}<\min _{k \neq j}\left|q_{j}-q_{k}\right|$ around $q_{j}$. Thus $\pi^{-1}\left(B_{r_{j}}^{3}\left(q_{j}\right)\right)$ is diffeomorphic to the punctured ball $B_{j}^{4} \backslash\{0\} \subset \mathbb{R}^{4}$ in a manner such that the $S^{1}$ action becomes the action of $S^{1} \subset \mathbb{C}$ on $\mathbb{C}^{2} \cong \mathbb{R}^{4}$ by scalar multiplication. We then define

$$
M_{V}:=\left(U_{V} \sqcup B_{1}^{4} \sqcup \cdots \sqcup B_{s}^{4}\right) / \sim
$$

where $\sim$ means that $B_{j}^{4} \backslash\{0\}$ is identified with $\pi^{-1}\left(B_{r_{j}}^{3}\left(q_{j}\right)\right)$. The map $\pi: U_{V} \rightarrow \mathbb{R}^{3} \backslash\left\{q_{1}, \ldots, q_{s}\right\}$ extends to a smooth map $\pi: M_{V} \rightarrow \mathbb{R}^{3}$. Note that there is an $S^{1}$ action on $M_{V}$ whose fixed points with index -1 are exactly the $q_{j}$ 's - called NUTs. If $\ell_{i j}$ is a straight line segment joining $q_{i}$ with $q_{j}$ then $\pi^{-1}\left(\ell_{i j}\right) \subset M_{V}$ is a smoothly embedded 2-sphere whose self-intersection number is -2 . The 2 -spheres $\pi^{-1}\left(\ell_{j, j+1}\right)$ with $j=1,2, \ldots, s-1$ are attached together according to the $A_{s-1}$ Dynkin diagram and generate the singular cohomology group $H^{2}\left(M_{V}, \mathbb{Z}\right) \cong \mathbb{Z}^{s-1}$. 
Take a real number $c>0$ and let $V: \mathbb{R}^{3} \backslash\left\{q_{1}, \ldots, q_{s}\right\} \rightarrow \mathbb{R}^{+}$be defined by

$$
V(x):=c+\frac{1}{2} \sum_{j=1}^{s} \frac{1}{\left|x-q_{j}\right|} .
$$

In this paper we will suppose $c=1$. Then $\triangle V=0$ that is, it is a positive harmonic function on $\mathbb{R}^{3} \backslash\left\{q_{1}, \ldots, q_{s}\right\}$. The cohomology class $\left[\frac{1}{2 \pi} *_{3} \mathrm{~d} V\right] \in H^{2}\left(U_{V}, \mathbb{Z}\right)$ is the first Chern class of the bundle $\pi: U_{V} \rightarrow \mathbb{R}^{3} \backslash\left\{q_{1}, \ldots, q_{s}\right\}$. Consequently there is a connection $\nabla$ on $\pi: U_{V} \rightarrow \mathbb{R}^{3} \backslash\left\{q_{1}, \ldots, q_{s}\right\}$ whose curvature is $*_{3} \mathrm{~d} V$. More precisely, let $\omega \in C^{\infty}\left(\Lambda^{1}\left(\mathbb{R}^{3} \backslash\left\{q_{1}, \ldots, q_{s}\right\}\right)\right)$ be a real valued connection 1-form on $\mathbb{R}^{3} \backslash\left\{q_{1}, \ldots, q_{s}\right\}$ so that $*_{3} \mathrm{~d} V=\frac{1}{\mathrm{i}} F_{\omega}=\mathrm{d} \omega$. The form $\omega$ is unique up to gauge transformation since $\mathbb{R}^{3} \backslash\left\{q_{1}, \ldots, q_{s}\right\}$ is simply connected. If we introduce a coordiante system $\left(x^{1}, x^{2}, x^{3}, \tau\right)$ where $x^{i}$ are Cartesian coordinates on $\mathbb{R}^{3}$ and $\tau \in[0,2 \pi)$ parameterizes the circles on $U_{V}$ then the multi-Taub-NUT metric $\left.g_{V}\right|_{U_{V}}$ on $U_{V}$ is defined to be

$$
\mathrm{d} s^{2}=V \pi^{*}\left(\left(\mathrm{~d} x^{1}\right)^{2}+\left(\mathrm{d} x^{2}\right)^{2}+\left(\mathrm{d} x^{3}\right)^{2}\right)+\frac{1}{V}\left(\mathrm{~d} \tau+\pi^{*} \omega\right)^{2}
$$

The space $\left(U_{V},\left.g_{V}\right|_{U_{V}}\right)$ extends smoothly across the fixed points of the $S^{1}$ action: write $V(x)=$ $V_{j}(x)+f(x)$ with $V_{j}(x):=\frac{1}{2\left|x-q_{j}\right|}$ and $f$ a smooth function around $q_{j}$. Let $\omega_{j}$ be the connection whose curvature is $*_{3} \mathrm{~d} V_{j}$ then we obtain a decomposition

$$
\mathrm{d} s^{2}=V_{j} \pi^{*}\left(\left(\mathrm{~d} x^{1}\right)^{2}+\left(\mathrm{d} x^{2}\right)^{2}+\left(\mathrm{d} x^{3}\right)^{2}\right)+\frac{1}{V_{j}}\left(\mathrm{~d} \tau+\pi^{*} \omega_{j}\right)^{2}+h
$$

where $h$ is a smooth symmetric bilinear form around $p_{j}$ induced by $f$. Moreover the first term is just the 1-Eguchi-Hanson metric on $\mathbb{R}^{4}$ which is known to be isometric to the standard flat metric on $\mathbb{R}^{4}$ hence extends over $p_{j}$.

We come therefore up with a Riemannian manifold $\left(M_{V}, g_{V}\right)$ with an isometric $S^{1}$ action whose fixed points with index -1 are $\pi^{-1}\left(q_{j}\right)$. It is moreover complete with a single ALF end, is anti-self-dual since $\mathrm{d} \omega=*_{3} \mathrm{~d} V$ that is, anti-half-conformally flat and Ricci-flat. Since $M_{V}$ is simply connected, it follows that it is hyper-Kähler as well i.e., there is an entire 2-sphere's worth of complex structures for which $g_{V}$ is a Kähler metric.

To display these complex structures explicitly at least on $U_{V}$, let $e_{1}, e_{2}, e_{3}$ be an orthonormal basis in $\mathbb{R}^{3}$. Consider these as constant vector fields on $\mathbb{R}^{3}$ and let $\hat{e}_{1}, \hat{e}_{2}, \hat{e}_{3}$ be their horizontal lifts to $U_{V}$ via the connection, as well as let $\hat{e}_{0}$ be the generator of the $S^{1}$-action. Then

$$
\left(\xi_{0}, \xi_{1}, \xi_{2}, \xi_{3}\right):=\left(\sqrt{V} \hat{e}_{0}, \frac{1}{\sqrt{V}} \hat{e}_{1}, \frac{1}{\sqrt{V}} \hat{e}_{2}, \frac{1}{\sqrt{V}} \hat{e}_{3}\right)
$$

is an orthonormal frame on $\left(U_{V}, g\right)$ which we define to be oriented. Relative to this frame we take the almost complex structure

$$
J_{e_{1}}:=\left(\begin{array}{cccc}
0 & -1 & 0 & 0 \\
1 & 0 & 0 & 0 \\
0 & 0 & 0 & -1 \\
0 & 0 & 1 & 0
\end{array}\right)
$$

It is parallel hence is integrable and it depends on the choice of $e_{1}$. Of course it extends over the whole $\left(M_{V}, g_{V}\right)$ which we continue to denote by $J_{e_{1}}$. By the aid of this picture we see 
that all possible orthogonal complex structures with compatible orientation on $\left(M_{V}, g_{V}\right)$ are parameterized by a $\mathbb{C} P^{1}$ and the situation can be described as a holomorphic fibration: the fiber over $e_{1} \in \mathbb{C} P^{1}$ is the complex manifold $\left(M_{V}, J_{e_{1}}\right)$ in accord with the general theory outlined in Sect. 2. Moreover if the direction of any of the straight line segments $\ell_{i j}$ coincides with $e_{1}$ then the corresponding 2-sphere $\pi^{-1}\left(\ell_{i j}\right)$ will represent a holomorphic curve with self-intersection number -2 in $\left(M_{V}, J_{e_{1}}\right)$. For a generic complex structure however, there are no holomorphic projective lines.

These complex structures on the whole $\left(M_{V}, g_{V}\right)$ can be described in a rather explicit algebraic way as follows (cf. [1, 28]).

Lemma 3.1. Fix a generic configuration of points $q_{1}, \ldots, q_{s}$ in $\mathbb{R}^{3}$ and consider the associated space $\left(M_{V}, g_{V}\right)$. Take a direction $e_{1}$ in $\mathbb{R}^{3}$. Assume that $e_{1}$ is not parallel with any line segments $\ell_{i j}$ joining $q_{i}$ with $q_{j}$. Then $\left(M_{V}, J_{e_{1}}\right)$ is biholomorphic to the algebraic surface $X \subset \mathbb{C}^{3}$ given by

$$
x y-\left(z-p_{1}\right) \ldots\left(z-p_{s}\right)=0
$$

with $(x, y, z) \in \mathbb{C}^{3}$ and fixed distinct complex numbers $p_{1}, \ldots, p_{s} \in \mathbb{C}$.

The $S^{1}$-action on $X$ is given by $(x, y, z, \tau) \mapsto\left(x \mathrm{e}^{\mathbf{i} \tau}, y \mathrm{e}^{-\mathbf{i} \tau}, z\right)$ with fixed points $\left(0,0, p_{j}\right) \in X$ of index -1 for all $j=1, \ldots, s$. Under this biholomorphism the fixed point $\pi_{V}^{-1}\left(q_{j}\right) \in M_{V}$ of the $S^{1}$ action on $M_{V}$ correspond to the fixed point $\left(0,0, p_{j}\right) \in X$ with the same index for all $j=1, \ldots, s$ consequently with this $S^{1}$-action on $X$ we obtain an isomorphism of complex manifolds with an $S^{1}$-action.

There exist at most $2\left(\begin{array}{l}s \\ 2\end{array}\right)=s(s-1)$ different directions when $e_{1}$ is parallel with any $\ell_{i j}$. In this case $\left(M_{V}, J_{e_{1}}\right)$ is biholomorphic to the complex surface $\widetilde{X}$ which arises by taking $p_{i}=p_{j}$ for fixed pair $(i, j)$ in (8) and blowing up the resulting singular surface $X^{*} \subset \mathbb{C}^{3}$ in $\left(0,0, p_{j}\right) \in X^{*}$.

As a real oriented four-manifold $\tilde{X}$ is diffeomorphic to $X$.

Proof. By genericity we assume that the straight line segments $\ell_{i j} \subset \mathbb{R}^{3}$ with $i, j=1,2, \ldots, s$ are mutually non-parallel. First take a direction $e_{1}$ in $\mathbb{R}^{3}$ which is not parallel with any $\ell_{i j}$. Let $\left(e_{2} e_{3}\right) \subset \mathbb{R}^{3}$ be the oriented plane passing through the origin, perpendicular to $e_{1}$ and identify it with the complex plane with its standard orientation: $\left(e_{2} e_{3}\right) \cong \mathbb{C}$ such that $e_{2} \mapsto 1$ and $e_{3} \mapsto$ i. Let $p_{e_{1}}: \mathbb{R}^{3} \rightarrow \mathbb{C}$ be the projection along $e_{1}$ which induces $P_{e_{1}}:=p_{e_{1}} \circ \pi: M_{V} \rightarrow \mathbb{C}$, the projection from $M_{V}$. Then $P_{e_{1}}$ is a holomorphic map from $\left(M_{V}, J_{e_{1}}\right)$ to $\mathbb{C}$. For a point $a \in M_{V}$ define a complex coordinate by $z:=P_{e_{1}}(a) \in \mathbb{C}$. In particular for a NUT $q_{j} \in \mathbb{R}^{3}$ put $p_{j}:=P_{e_{1}}\left(\pi^{-1}\left(q_{j}\right)\right)=p_{e_{1}}\left(q_{j}\right) \in \mathbb{C}$ for all $j=1, \ldots, s$ and also call them NUTs.

Next we analyze the preimage $P_{e_{1}}^{-1}(z) \subset M_{V}$. If $z \neq p_{j} \in \mathbb{C}$ then $P_{e_{1}}^{-1}(z)$ is holomorphically isomorphic to an infinite cylinder $\mathbb{C}^{*}:=\left\{(x, y) \in \mathbb{C}^{2} \mid x y=1\right\}$ in $\left(M_{V}, J_{e_{1}}\right)$, since the directions $\hat{e}_{0}$ and $\hat{e}_{1}$ span a holomorphic line for the complex structure $J_{e_{1}}$. On the other hand $P_{e_{1}}^{-1}\left(p_{j}\right)$ is homeomorphic to two complex affine lines intersecting in one point. In particular we have a model like $\left\{(x, y) \in \mathbb{C}^{2} \mid x y=0\right\}$ for $P_{e_{1}}^{-1}\left(p_{j}\right)$. Since the complex structure on the fibers is independent of $z$, the above two cases can be managed together into a global equation by writing $x y=c(z)$ where $c(z)$ is a polynomial in the variable $z$ on $\mathbb{C}$ which vanishes exactly in the points $p_{1}, \ldots, p_{s} \in \mathbb{C}$. Therefore we put

$$
c(z):=\left(z-p_{1}\right) \ldots\left(z-p_{s}\right)
$$

to obtain the desired biholomorphism between $\left(M_{V}, J_{e_{1}}\right)$ and $X$ given by (8).

The statement about the $S^{1}$-action is clear. 
If $e_{1}$ happens to be parallel with any $\ell_{i j}$ connecting $q_{i} \in \mathbb{R}^{3}$ and $q_{j} \in \mathbb{R}^{3}$ then we find $p_{e_{1}}\left(q_{i}\right)=p_{i}=p_{e_{1}}\left(q_{j}\right)=p_{j} \in \mathbb{C}$ hence two roots coincide in (8). However now $P_{e_{1}}^{-1}\left(p_{i}\right)=P_{e_{1}}^{-1}\left(p_{j}\right)$ consists of not only two complex affine lines as above but in addition the projective line $\pi^{-1}\left(\ell_{i j}\right)$ and the two complex affine lines do not intersect each other rather each individual line hits $\pi^{-1}\left(\ell_{i j}\right)$ in one point respectively. These two points are distinct. The situation can be modeled on $\left\{(x, y,[u: v]) \in \mathbb{C}^{2} \times \mathbb{C} P^{1} \mid x y=0, x v=y u\right\}$. Therefore to obtain the resulting smooth complex surface we have to take a singular curve $X^{*} \subset \mathbb{C}^{3}$ represented by (8) with $p_{i}=p_{j}$ and blow it up at $\left(0,0, p_{i}\right)=\left(0,0, p_{j}\right) \in X^{*}$ to obtain $\tilde{X}$.

It readily follows that as a real oriented four-manifold, $\widetilde{X}$ is diffeomorphic to $X$ since both are just the original $M_{V}$ equipped with different complex structures. $\diamond$

Remark. Incidentally we note that the above definition of the coordinate $z$ depends on the identification of the oriented plane perpendicular to $e_{1}$ with $\mathbb{C}$, i.e. on the choice of a vector $e_{2}$ in the tangent space of $\mathbb{C} P^{1}$ at $e_{1}$. For all such choices, the above procedure yields a complex number. As $T \mathbb{C} P^{1} \cong H^{2}$, this means that globally over $\mathbb{C} P^{1}$ the coordinate $z$ must be regarded as a section of the line bundle $H^{2}$. This fact will play an important role in constructing the twistor space, cf. Sect. 5 .

\section{Moduli spaces with trivial holonomy}

We already know that a multi-Taub-NUT space $\left(M_{V}, g_{V}\right)$ is anti-half-conformally flat and Ricciflat. Consequently for its curvature $W_{g_{V}}^{+}=0$ and $s_{g_{V}}=0$ holds and the Atiyah-Hitchin-Singer theorem applies and we can construct $\mathrm{SU}(2)^{+}$(anti-)self-dual connections on the (trivial) positive chiral spinor bundle $\Sigma^{+}$. If we fix an orientation coming from any compex structure in the hyperKähler family, these connections will be anti-self-dual. As we have seen in Section 2, this approach amounts to find positive solutions of the Laplace equation $\triangle_{g_{V}} f=0$ with a finite number of mild singularities and appropriate bounds at infinity over the original manifold $\left(M_{V}, g_{V}\right)$. This program partially was carried out in $[12,10]$ and will be stretched to its limits here.

An abundance of such harmonic functions are provided by minimal positive Green functions therefore we make a short digression on them following [17, 21, 34].

Definition 4.1. Let $(M, g)$ be a non-compact complete Riemannian manifold of dimension $m \geq 2$ and $y \in M$ be a point. A function $G(\cdot, y): M \backslash\{y\} \rightarrow \mathbb{R}$ is called the minimal positive Green function concentrated at $y \in M$ if it has the following properties:

(i) It satisfies $\triangle_{g} G(\cdot, y)=\delta_{y}$ in the sense of distributions on $(M, g)$;

(ii) Let $r:=d(\cdot, y)$ denote the distance from $y$ on $(M, g)$. If $r \rightarrow+\infty$ then $G(\cdot, y)$ tends to zero and if $r \rightarrow 0$ then $G(\cdot, y)$ is $O\left(r^{2-m}\right)$ and $\left|\nabla_{y} G(\cdot, y)\right|_{g}$ is $O\left(r^{1-m}\right)$ for $m \geq 3$ as well as $G(\cdot, y)$ is $O(\log r)$ and $\left|\nabla_{y} G(\cdot, y)\right|_{g}$ is $O\left(r^{-1}\right)$ for $m=2$;

(iii) Moreover $0<G(\cdot, y)$ and $G(\cdot, y) \leq G^{\prime}(\cdot, y)$ for any other positive Green function with the same singularity.

This function if exists is obviously unique and is characterized by these properties. 
One can try to construct them as follows [21, p. 229]. Take $y \in M$ and $\Omega \Subset M$ an open subset with smooth boundary and $\bar{\Omega}$ compact such that $y \in \Omega$. Let $G_{\Omega}(\cdot, y)$ be the unique Green function on $\Omega$ satisfying

$$
\triangle_{g} G_{\Omega}(\cdot, y)=\delta_{y} \quad \text { and }\left.\quad G_{\Omega}(\cdot, y)\right|_{\partial \bar{\Omega}}=0 .
$$

By the maximum principle we can assume that $G_{\Omega}(\cdot, y)$ is positive on $\Omega \backslash\{y\}$ and, if we extend $G_{\Omega}(\cdot, y)$ by zero outside $\Omega$, then $G_{\Omega}(\cdot, y) \leq G_{\Omega^{\prime}}(\cdot, y)$ whenever $\Omega \subseteq \Omega^{\prime}$. Consequently setting

$$
G(\cdot, y):=\sup _{\Omega} G_{\Omega}(\cdot, y)
$$

it follows that if this function exists then it is the minimal positive Green function with singularity in $y \in M$. One can prove [21, Theorem 8.1] (also cf. [17, 34]) that minimal positive Green functions with singularity $y \in M$ constructed this way either exist over $M$ for all $y$ or do not exist at all. In the former case $(M, g)$ is called non-parabolic, while in the later case called parabolic.

Therefore we should be able to decide whether or not a Riemannian manifold is parabolic. For our purposes the easiest way is to recall a result of Varopoulos [34] which states that if the Ricci curvature of a non-compact complete Riemannian manifold $(M, g)$ with $m \geq 2$ is non-negative and for some point $x \in M$ with its geodesic ball of radius $r$

$$
\int_{1}^{+\infty} \frac{r}{\operatorname{Vol}_{g}\left(B_{r}^{4}(x)\right)} \mathrm{d} r<+\infty
$$

holds then $(M, g)$ is non-parabolic.

With this in mind for the Ricci-flat multi-Taub-NUT space $\left(M_{V}, g_{V}\right)$ we find that

$$
\operatorname{Vol}_{g_{V}}\left(B_{r}^{4}(x)\right) \sim \frac{8 \pi^{2}}{3} r^{3}
$$

demonstrating that it is non-parabolic (and we can also see that it is indeed ALF); consequently for all $y \in M_{V}$ an associated minimal positive Green function $G(\cdot, y)$ exists. In fact they were explicitly constructed by Page [31] and the simplest of them also appeared in [12]. Making use of these functions we obtain plenty of positive harmonic functions of the form

$$
f_{y, \lambda}(x):=1+\lambda G(x, y)
$$

with a real constant $\lambda \in(0,+\infty)$.

We claim that these functions have the required properties and can be used to construct finite-energy smooth $\mathrm{SU}(2)^{+}$anti-instantons over the multi-Taub-NUT space. To check this, we construct the associated five parameter family $\nabla_{\tilde{A}_{y, \lambda}}$ of non-gauge equivalent anti-self-dual $\mathrm{SU}(2)^{+}$connections on $\Sigma^{+}$with respect to the orientation on $\left(M_{V}, g_{V}\right)$ coming from the complex structures and demonstrate first that they are smooth around $y \in M_{V}$ hence everywhere and secondly that they are admissible hence in particular have finite energy.

Lemma 4.1. Fix a point $y \in M_{V}$ and a number $\lambda \in(0,+\infty)$. Take a geodesic ball $B_{\varepsilon}^{4}(y) \subset M_{V}$ around $y$ and write $B_{\varepsilon}^{*}(y):=B_{\varepsilon}^{4}(y) \backslash\{y\}$ for the punctured ball. Consider the orthonormal frame (7) on $\left(B_{\varepsilon}^{4}(y), g_{V}\right)$. In this gauge plugging (9) into (2) write $\left.\nabla_{\tilde{A}_{y, \lambda}}\right|_{B_{\varepsilon}^{*}(y)}=\mathrm{d}+\tilde{A}_{y, \lambda}$ for the resulting anti-self-dual connection. 
There exists an $L_{1, \tilde{A}_{y, \lambda}}^{2}$ gauge transformation $\gamma: B_{\varepsilon}^{*}(y) \rightarrow \mathrm{SU}(2)^{+}$such that the gauge transformed potential $\tilde{A}_{y, \lambda}^{\prime}:=\gamma^{-1} \tilde{A}_{y, \lambda} \gamma+\gamma^{-1} \mathrm{~d} \gamma$ extends smoothly over $B_{\varepsilon}^{4}(y)$ that is the $\mathrm{SU}(2)^{+}$ connection $\left.\nabla_{\tilde{A}_{y, \lambda}}\right|_{B_{\varepsilon}^{4}(y)}$ is smooth on $\left.\Sigma^{+}\right|_{B_{\varepsilon}^{4}(y)}$.

Consequently the anti-self-dual connetion $\nabla_{\tilde{A}_{y, \lambda}}$ is smooth everywhere on $\Sigma^{+}$.

Proof. Take an orthonormal frame $\left(\xi^{0}, \xi^{1}, \xi^{2}, \xi^{3}\right)$ over $B_{\varepsilon}^{4}(y)$ as in (7) with its associated complex structure $J_{e_{1}}$ on $M_{V}$. Since the multi-Taub-NUT metric is Kähler with respect to this complex structure, there exists a complex coordinate system $\left(z^{1}, z^{2}\right)$ on $B_{\varepsilon}^{4}(y)$ centered in $y$ such that

$$
\left.g_{V}\right|_{B_{\varepsilon}^{4}(y)}=\left(\delta_{i j}+O\left(r^{2}\right)\right) \mathrm{d} z^{i} \mathrm{~d} \bar{z}^{j}
$$

i.e., the metric osculates the flat metric in second order. Take a neighbourhood $y \in U \subset M_{V}$ and identify it with $\mathbb{H}$ such that $y \in M_{V}$ is mapped into $\mathbf{y}:=y^{0}+y^{1} \mathbf{i}+y^{2} \mathbf{j}+y^{3} \mathbf{k} \in \mathbb{H}$. Then $B_{\varepsilon}^{4}(y) \subset U$ becomes a quaternionic ball $B_{\varepsilon}^{4}(\mathbf{y}) \subset \mathbb{H}$ centered about $\mathbf{y}$. Introduce a quaternionic coordinate $\mathbf{x} \in \mathbb{H}$ via $\mathbf{x}:=\mathbf{y}+z^{1}+z^{2} \mathbf{j}$ on it. To simplify notation also write $g_{V}$ for the pullback metric on $B_{\varepsilon}^{4}(\mathbf{y})$. There is a bounded positive function $a$ such that $r(\mathbf{x})=a(\mathbf{x})|\mathbf{x}-\mathbf{y}|$ hence setting $z^{1}=x^{0}+x^{1} \mathbf{i}$ and $z^{2}=x^{2}+x^{3} \mathbf{i}$ we find in this real coordinate system that

$$
\xi^{i}(\mathbf{x})=\left(\delta_{j}^{i}+O\left(|\mathbf{x}-\mathbf{y}|^{2}\right)\right) \mathrm{d} x^{j} \quad \text { and } \quad \xi_{i}(\mathbf{x})=\left(\delta_{i}^{j}+O\left(|\mathbf{x}-\mathbf{y}|^{2}\right) \frac{\partial}{\partial x^{j}} .\right.
$$

Similarly for the harmonic function in (9) we obtain

$f_{\mathbf{y}, \lambda}(\mathbf{x})=1+\frac{\lambda}{|\mathbf{x}-\mathbf{y}|^{2}}+O\left(|\mathbf{x}-\mathbf{y}|^{-1}\right) \quad$ and $\quad \mathrm{d} f_{\mathbf{y}, \lambda}(\mathbf{x})=-2 \lambda\left(\frac{x^{i}-y^{i}}{|\mathbf{x}-\mathbf{y}|^{4}} \delta_{j}^{i}+O\left(|\mathbf{x}-\mathbf{y}|^{-2}\right)\right) \mathrm{d} x^{j}$

Inserting these expansions into (3) we obtain $a_{\mathbf{y}, \lambda}=B_{\mathbf{y}, \lambda}+b_{\mathbf{y}, \lambda}$ for the perturbation where the singular Euclidean term looks like

$$
B_{\mathbf{y}, \lambda}(\mathbf{x})=\operatorname{Im} \frac{\lambda(\overline{\mathbf{x}}-\overline{\mathbf{y}}) \mathrm{d} \mathbf{x}}{|\mathbf{x}-\mathbf{y}|^{2}\left(\lambda+|\mathbf{x}-\mathbf{y}|^{2}\right)}
$$

and $b_{\mathbf{y}, \lambda}$ is of $O(1)$ hence regular in $B_{\varepsilon}^{4}(\mathbf{y})$.

Regarding the spin connection $\nabla_{A}$, note that the $\mathrm{SU}(2)^{+}$bundle $\Sigma^{+}$is trivial over $M_{V}$. The metric is anti-self-dual hence the spin connection is flat and since $M_{V}$ is simply connected it is just the trivial flat connection. Writing $\left.\nabla_{A}\right|_{B_{\varepsilon}^{4}(y)}=\mathrm{d}+A$ we simply find $A=0$ in the natural gauge (7).

Putting all of these into (2) we eventually come up in the gauge (7) we use with an expansion of the vector potential as follows:

$$
\tilde{A}_{\mathbf{y}, \lambda}=B_{\mathbf{y}, \lambda}+b_{\mathbf{y}, \lambda}
$$

whose only singular term is $B_{\mathbf{y}, \lambda}$ but still $\tilde{A}_{\mathbf{y}, \lambda} \in L_{1, \tilde{A}_{\mathbf{y}, \lambda}}^{2}\left(\left.\Lambda^{1} B_{\varepsilon}^{*}(\mathbf{y}) \otimes \operatorname{End} \Sigma^{+}\right|_{B_{\varepsilon}^{*}(\mathbf{y})}\right)$.

Calculating the curvature we find

$$
F_{\tilde{A}_{\mathbf{y}, \lambda}}=F_{B_{\mathbf{y}, \lambda}}+\mathrm{d}_{B_{\mathbf{y}, \lambda}} b_{\mathbf{y}, \lambda}+b_{\mathbf{y}, \lambda} \wedge b_{\mathbf{y}, \lambda}
$$

that is,

$$
F_{\tilde{A}_{\mathbf{y}, \lambda}}(\mathbf{x})=\frac{\lambda \mathrm{d} \overline{\mathbf{x}} \wedge \mathrm{d} \mathbf{x}}{\left(\lambda+|\mathbf{x}-\mathbf{y}|^{2}\right)^{2}}+\mathrm{d}_{B_{\mathbf{y}, \lambda}(\mathbf{x})} b_{\mathbf{y}, \lambda}(\mathbf{x})+b_{\mathbf{y}, \lambda}(\mathbf{x}) \wedge b_{\mathbf{y}, \lambda}(\mathbf{x})
$$


demonstrating that the curvature $F_{B_{\mathbf{y}, \lambda}}$ of the Euclidean term as well as $b_{\mathbf{y}, \lambda} \wedge b_{\mathbf{y}, \lambda}$ are smooth in the ball $B_{\varepsilon}^{4}(\mathbf{y})$ meanwhile $\mathrm{d}_{B_{\mathbf{y}, \lambda}} b_{\mathbf{y}, \lambda}$ is singular. Let $\langle\cdot, \cdot\rangle$ denote the pointwise scalar product and $|\cdot|$ the induced pointwise norm on $\mathfrak{s u}(2)^{+}$-valued 2-forms associated to the Killing norm on $\mathfrak{s u}(2)^{+}$and the metric on $\Lambda^{2} M_{V}$. This gives rise to the scalar product $(\cdot, \cdot)_{L^{2}}$ and norm $\|\cdot\|_{L^{2}}$ on the punctured ball. Separating the regular and singular terms we obtain

$$
\begin{aligned}
\left\|F_{\tilde{A}_{\mathbf{y}, \lambda}}\right\|_{L^{2}\left(B_{\varepsilon}^{*}(\mathbf{y})\right)}^{2} & =\left\|F_{B_{\mathbf{y}, \lambda}}+b_{\mathbf{y}, \lambda} \wedge b_{\mathbf{y}, \lambda}\right\|_{L^{2}\left(B_{\varepsilon}^{*}(\mathbf{y})\right)}^{2}+\left\|\mathrm{d}_{B_{\mathbf{y}, \lambda}} b_{\mathbf{y}, \lambda}\right\|_{L^{2}\left(B_{\varepsilon}^{*}(\mathbf{y})\right)}^{2} \\
& +2\left(F_{B_{\mathbf{y}, \lambda}}+b_{\mathbf{y}, \lambda} \wedge b_{\mathbf{y}, \lambda}, \mathrm{d}_{B_{\mathbf{y}, \lambda}} b_{\mathbf{y}, \lambda}\right)_{L^{2}\left(B_{\varepsilon}^{*}(\mathbf{y})\right)} .
\end{aligned}
$$

Since the dual of the volume form $*\left(\left.\mathrm{~d} V_{g_{V}}\right|_{B_{\varepsilon}(\mathbf{y})}(\mathbf{x})\right)$ with respect to the flat Hodge star $*$ on $\mathbb{H}$ is $O\left(|\mathbf{x}-\mathbf{y}|^{3}\right)$ we find on the one hand that the regular energy term is of course finite:

$$
\left\|F_{B_{\mathbf{y}, \lambda}}+b_{\mathbf{y}, \lambda} \wedge b_{\mathbf{y}, \lambda}\right\|_{L^{2}\left(B_{\varepsilon}^{*}(\mathbf{y})\right)}^{2}=\int_{B_{\varepsilon}^{*}(\mathbf{y})}\left|F_{B_{\mathbf{y}, \lambda}}+b_{\mathbf{y}, \lambda} \wedge b_{\mathbf{y}, \lambda}\right|^{2} \mathrm{~d} V_{g_{V}} \leq c_{3} \int_{0}^{\varepsilon} r^{3} \mathrm{~d} r<+\infty .
$$

The singular term $\left|\mathrm{d}_{B_{\mathbf{y}, \lambda}(\mathbf{x})} b_{\mathbf{y}, \lambda}(\mathbf{x})\right|$ on the other hand is $O\left(|\mathbf{x}-\mathbf{y}|^{-1}\right)$ consequently

$$
\left\|\mathrm{d}_{B_{\mathbf{y}, \lambda}} b_{\mathbf{y}, \lambda}\right\|_{L^{2}\left(B_{\varepsilon}^{*}(\mathbf{y})\right)}^{2}=\int_{B_{\varepsilon}^{*}(\mathbf{y})}\left|\mathrm{d}_{B_{\mathbf{y}, \lambda}} b_{\mathbf{y}, \lambda}\right|^{2} \mathrm{~d} V_{g_{V}} \leq c_{4} \int_{0}^{\varepsilon} r^{-2} r^{3} \mathrm{~d} r<+\infty .
$$

Finally, the cross term also satisfies the estimate (here and only here $|\cdot|$ is the ordinary pointwise absolute value on reals)

$$
\begin{aligned}
\left|\left(F_{B_{\mathbf{y}, \lambda}}+b_{\mathbf{y}, \lambda} \wedge b_{\mathbf{y}, \lambda}, \mathrm{d}_{B_{\mathbf{y}, \lambda}} b_{\mathbf{y}, \lambda}\right)_{L^{2}\left(B_{\varepsilon}^{*}(\mathbf{y})\right)}\right| & \leq \int_{B_{\varepsilon}^{*}(\mathbf{y})}\left|\left\langle F_{B_{\mathbf{y}, \lambda}}+b_{\mathbf{y}, \lambda} \wedge b_{\mathbf{y}, \lambda}, \mathrm{d}_{B_{\mathbf{y}, \lambda}} b_{\mathbf{y}, \lambda}\right\rangle\right| \mathrm{d} V_{g_{V}} \\
& \leq c_{5} \int_{0}^{\varepsilon} r^{-1} r^{3} \mathrm{~d} r<+\infty .
\end{aligned}
$$

We conclude that the last two conditions in (5) hold hence the apparent singularity in $\nabla_{\tilde{A}_{y, \lambda}}$ at $y$ can be removed by a gauge transformation via Uhlenbeck's theorem [33] providing us with a smooth anti-self-dual SU $(2)^{+}$connection on the whole positive spinor bundle $\Sigma^{+}$.

Proceeding further we demonstrate that our solutions are anti-instantons, i.e. have finite energy. To carry out this we construct an asymptotic expansion for (9) to see explicitly its fall-off. Intuitively [10], a harmonic function with a fixed singularity on $\left(M_{V}, g_{V}\right)$ asymptotically looks like pulling the singular point of the function as well as all the NUTs in $M_{V}$ together. Therefore our strategy will be as follows: we construct an expansion of a harmonic function whose singularity coincides with that of a singular multi-Taub-NUT space. After finding its asymptotic expansion we show that the decay rate of the leading term remains unchanged if we perturb this singular space into the original smooth one.

In light of Lemma 3.1 this collapsed space arises when all the roots of the right hand side in (8) coincide: $p_{1}=\cdots=p_{s}=0$ hence the resulting space $X^{*} \subset \mathbb{C}^{3}$ is given by $x y-z^{s}=0$. On the other hand, if $(u, v) \in \mathbb{C}^{2}$ then the polynomials $u^{s}, v^{s}$ and $u v$ are invariant under the 
standard action of $\mathbb{Z}_{s} \subset \mathrm{SU}(2)$ on $\mathbb{C}^{2}$ and putting $x:=u^{s}, y:=v^{s}, z:=u v$ then they also satisfy $x y-z^{s}=0$ i.e., $X^{*}$ is a model in $\mathbb{C}^{3}$ for the $A_{s-1}$-singularity $M_{V}^{*}:=\mathbb{C}^{2} / \mathbb{Z}_{s}$.

Removing the origin $\{0\} \in \mathbb{C}^{2}$ the resulting smooth space $W^{*}:=\mathbb{C}^{2} \backslash\{0\} / \mathbb{Z}_{s}$ is the neck at infinity and is topologically a lens space: $W^{*} \cong L(s,-1) \times \mathbb{R}^{+}$. Here $L(s,-1) \cong S^{3} / \mathbb{Z}_{s}$ denotes the usual lens space of $(s,-1)$-type with its orientation inherited from the complex structure on $W^{*}$. Regarding $W^{*}$ as a complex line bundle of Chern class $-s$ over $S^{2}$ let us denote by $(r, \tau)$ polar coordinates on the fibres centered in the singular origin of $\mathbb{C}^{2} / \mathbb{Z}_{s}$ and by $(\Theta, \phi)$ the usual spherical coordinates on the base. ${ }^{2}$ We simply get

$$
V(r)=1+\frac{s}{2 r}, \quad \alpha(\Theta, \phi)=\frac{s}{2} \cos \Theta \mathrm{d} \phi
$$

consequently the multi-Taub-NUT metric (6) reduces to a singular metric $g_{V}^{*}$ on $M_{V}^{*}$ whose shape on $W^{*}$ is

$$
\mathrm{d} s^{2}=\frac{2 r+s}{2 r}\left(\mathrm{~d} r^{2}+r^{2} \mathrm{~d} \Theta^{2}+r^{2} \sin ^{2} \Theta \mathrm{d} \phi^{2}\right)+\frac{s^{2}}{4} \frac{2 r}{2 r+s}(\mathrm{~d} \tau+\cos \Theta \mathrm{d} \phi)^{2}
$$

with parameters

$$
0<r<+\infty, \quad 0 \leq \tau<\frac{4 \pi}{s}, \quad 0 \leq \phi<2 \pi, \quad 0 \leq \Theta<\pi .
$$

One easily calculates $\operatorname{det} g_{V}^{*}=\frac{s^{2}}{4}\left(\frac{2 r+s}{2}\right)^{2} r^{2} \sin ^{2} \Theta$ and by referring to the local expression for the Laplacian of any metric

$$
\triangle_{g}=\sum_{i, j} \frac{1}{\sqrt{\operatorname{det} g}} \frac{\partial}{\partial x^{i}}\left(\sqrt{\operatorname{det} g} g^{i j} \frac{\partial}{\partial x^{j}}\right)
$$

where $g^{i j}$ are the components of the inverse matrix, our singular Laplacian looks like

$$
\begin{aligned}
\triangle_{g_{V}^{*}} & =\frac{2 r}{2 r+s} \frac{\partial^{2}}{\partial r^{2}}+\frac{4}{2 r+s} \frac{\partial}{\partial r}+\frac{2(2 r+s)^{2} \sin ^{2} \Theta+2 s^{2} \cos ^{2} \Theta}{r s^{2}(2 r+s) \sin ^{2} \Theta} \frac{\partial^{2}}{\partial \tau^{2}} \\
& +\frac{2}{r(2 r+s)}\left(\frac{\partial^{2}}{\partial \Theta^{2}}+\cot \Theta \frac{\partial}{\partial \Theta}\right)+\frac{2}{r(2 r+s) \sin ^{2} \Theta}\left(\frac{\partial^{2}}{\partial \phi^{2}}-2 \cos \Theta \frac{\partial^{2}}{\partial \tau \partial \phi}\right) .
\end{aligned}
$$

This apparently new Laplacian can be re-expressed in old terms as follows. The lens space with its standard metric induced from the round $S^{3}$ has an associated Laplacian

$$
\triangle_{L(s,-1)}=\frac{\partial^{2}}{\partial \Theta^{2}}+\cot \Theta \frac{\partial}{\partial \Theta}+\frac{1}{\sin ^{2} \Theta}\left(\frac{\partial^{2}}{\partial \phi^{2}}-2 \cos \Theta \frac{\partial^{2}}{\partial \tau \partial \phi}+\frac{\partial^{2}}{\partial \tau^{2}}\right)
$$

in Euler coordinates. By the aid of this we plainly obtain

$$
V(r) \triangle_{g_{V}^{*}}=\frac{\partial^{2}}{\partial r^{2}}+\frac{2}{r} \frac{\partial}{\partial r}+\left(\frac{4}{r s}+\frac{4}{s^{2}}\right) \frac{\partial^{2}}{\partial \tau^{2}}+\frac{1}{r^{2}} \triangle_{L(s,-1)} .
$$

As we have seen, we have to find positive solutions to $\triangle_{g_{V}^{*}} f^{*}=0$ or equivalently to

$$
V \triangle_{g_{V}^{*}} f^{*}=0
$$

\footnotetext{
${ }^{2}$ In other words $(\tau, \Theta, \phi)$ are Euler coordinates on the lens space and $r$ is the radial coordiante on the cone $\mathbb{C}^{2} / \mathbb{Z}_{s}$. For clarity we mention that $r$ defined this way asymptotically accords with the distance function $r$ used in Definition 4.1 while $\tau$ here is $\frac{2}{s}$-times the periodic coordinate $\tau$ appeared in (6).
} 
with singularity in the origin $r=0$. These functions can be constructed easily by separating variables. To this end we introduce an orthonormal system of bounded smooth functions on the lens space $L(s,-1)$ by the (three dimensional) spherical harmonics $Y_{j}^{k, l}$ with $j=0,1, \ldots$ and $-j \leq k \leq j,-\left[\frac{2 j}{s}\right] \leq l \leq\left[\frac{2 j}{s}\right]$ (for a fixed $j$, there are $(2 j+1)\left(\left[\frac{2 j}{s}\right]+1\right)$ independent spherical harmonics on $L(s,-1))$. In Euler coordinates these take the shape

$$
Y_{j}^{k, l}(\tau, \phi, \Theta)=N_{j}^{k, l, s} \mathrm{e}^{\mathrm{i}\left(\frac{l s}{2} \tau+k \phi\right)} P_{j}^{k, l}(\cos \Theta)
$$

with appropriate normalizing constants $N_{j}^{k, l, s}$. The generalized associated Legendre polynomial $P_{j}^{k, l}(x)$ is the unique real analytic solution on $[-1,1]$ of the following differential equation:

$$
\left[\left(1-x^{2}\right) \frac{\mathrm{d}^{2}}{\mathrm{~d} x^{2}}-2 x \frac{\mathrm{d}}{\mathrm{d} x}-\frac{k^{2}-x(k l s)+\frac{l^{2} s^{2}}{4}}{1-x^{2}}+j(j+1)\right] P_{j}^{k, l}(x)=0 .
$$

Note that taking $l=0$ the function $P_{j}^{k, 0}(x)$ reduces to the standard associated Legendre polynomial $P_{j}^{k}(x)$ as well as $Y_{j}^{k, 0}(\tau, \phi, \Theta)$ gives back the usual spherical harmonics $Y_{j}^{k}(\phi, \Theta)$ on $S^{2}$. The spherical harmonics obey

$$
\frac{\partial Y_{j}^{k, l}}{\partial \tau}=\frac{\mathbf{i} l s}{2} Y_{j}^{k, l} \quad \text { and } \quad \triangle_{L(s,-1)} Y_{j}^{k, l}=-j(j+1) Y_{j}^{k, l} .
$$

In this basis we seek solutions in the form

$$
f^{*}(r, \tau, \phi, \Theta)=\sum_{j=0}^{\infty} \sum_{k=-j}^{j} \sum_{l=-\left[\frac{2 j}{s}\right]}^{\left[\frac{2 j}{s}\right]} \lambda_{j}^{k, l} \varrho_{j}^{k, l}(r) Y_{j}^{k, l}(\tau, \phi, \Theta)
$$

where $\lambda_{j}^{k, l}$ are complex numbers and $\varrho_{j}^{k, l}$ are radial functions. Inserting this ansatz into (11) and then exploiting the eigenfunction properties of the spherical harmonics our equation cuts down to a family of ordinary differential equations

$$
\left[\frac{\mathrm{d}^{2}}{\mathrm{~d} r^{2}}+\frac{2}{r} \frac{\mathrm{d}}{\mathrm{d} r}-\frac{j(j+1)}{r^{2}}-\frac{s l^{2}}{r}-l^{2}\right] \varrho_{j}^{k, l}(r)=0
$$

with $j=0,1, \ldots$ and $-j \leq k \leq j,-\left[\frac{2 j}{s}\right] \leq l \leq\left[\frac{2 j}{s}\right]$. The functions $\varrho_{j}^{k, l}$ are in fact independent of the index $k$ due to the $\mathrm{U}(2)$ symmetry of the space $\left(M_{V}^{*}, g_{V}^{*}\right)$. Two particular solutions if $l=0$ are

$$
K_{-j-1}^{0}(r)=r^{-j-1} \quad \text { and } \quad K_{j}^{0}(r)=r^{j}
$$

moreover for $l>0$ and $r \ll 1$ we know that

$$
K_{-j-1}^{l}(r)=r^{-j-1}(1+O(r)) \quad \text { and } \quad K_{j}^{l}(r)=r^{j}(1+O(r))
$$

meanwhile for $l>0$ and $r \gg 1$ we obtain

$$
K_{-j-1}^{l}(r)=\mathrm{e}^{-l r} r^{-\frac{s l}{2}-1}\left(1+O\left(r^{-1}\right)\right) \quad \text { and } \quad K_{j}^{l}(r)=\mathrm{e}^{l r} r^{\frac{s l}{2}-1}\left(1+O\left(r^{-1}\right)\right) .
$$

Separating variables therefore forces us to put the pointlike singularity of $f^{*}$ into the origin of $M_{V}^{*}$ showing our approach is consistent. To summarize, the general solution formally looks like

$$
f^{*}(r, \tau, \phi, \Theta)=\sum_{j=0}^{\infty} \sum_{k=-j}^{j} \sum_{l=-\left[\frac{2 j}{s}\right]}^{\left[\frac{2 j}{s}\right]}\left(\lambda_{j}^{k, l} K_{-j-1}^{l}(r)+\mu_{j}^{k, l} K_{j}^{l}(r)\right) Y_{j}^{k, l}(\tau, \phi, \Theta)
$$


with the only singularity in the origin.

Because $\overline{Y_{j}^{k, l}}=Y_{j}^{-k,-l}$, a real basis for the solutions is given by

$$
\frac{1}{2}\left(Y_{j}^{k, l}+Y_{j}^{-k,-l}\right) \quad \text { and } \quad \frac{1}{2 \mathbf{i}}\left(Y_{j}^{k, l}-Y_{j}^{-k,-l}\right) .
$$

Moreover from (12) we obtain that $K_{-j-1}^{l}=K_{-j-1}^{-l}$ and $K_{j}^{l}=K_{j}^{-l}$ consequently if we suppose $\overline{\lambda_{j}^{k, l}}=\lambda_{j}^{-k,-l}$ as well as $\overline{\mu_{j}^{k, l}}=\mu_{j}^{-k,-l}$ then we come up with formal real solutions in (13). In addition asymptotically bounded solutions from (13) arise by setting $\mu_{j}^{k, l}=0$ for $j>0$.

Taking into account that $Y_{0}^{0,0}$ is a constant, the leading $j=0$ term shows that the asymptotic shape of asymptotically bounded real harmonic functions looks like $f^{*}(r)=\mu+\lambda r^{-1}+O\left(r^{-2}\right)$ (we put simply $\mu_{0}^{0,0}=\mu$ and $\lambda_{0}^{0,0}=\lambda$, both real) on the singular space $\left(M_{V}^{*}, g_{V}^{*}\right.$ ).

Now we ask ourselves how this picture changes over the original non-singular space $\left(M_{V}, g_{V}\right)$ for the Green function $G(\cdot, y)$ with singularity at $y \in M_{V}$. For this purpose, we use results of [30], where a careful study of analytic properties of the Laplace operator on perturbations of ALF spaces has been carried out. If $W^{*}$ and $W$ are the necks of $M_{V}^{*}$ and $M_{V}$ respectively then we suppose $W^{*} \cong W$ and use the same coordinate system along them. It is clear that for $\alpha=2$ we have $\left.g_{V}\right|_{W}=\left.g_{V}^{*}\right|_{W^{*}}+O\left(r^{-\alpha}\right) h$ with some $O(1)$ symmetric tensor field $h$. Let $\chi$ be a cut-off function supported in a compact neighborhood of $y$ and identically equal to 1 in a smaller neighborhood of $y$. We will try to look for the Green function in the form

$$
G(\cdot, y)=\chi G^{(4)}(\cdot, y)+u
$$

where $G^{(4)}(\cdot, y)$ stands for the Green function on $\mathbb{R}^{4}$ and $u$ is some locally $L^{2}$ function. Then, $u$ must satisfy the equation

$$
\triangle_{g_{V}} u=f
$$

for some prescribed compactly supported smooth function $f$ only depending on $\chi$ and $G^{(4)}(\cdot, y)$. In particular, $f$ belongs to any weighted Sobolev space $L_{\delta}^{2}\left(M_{V} ; \mathbb{R}\right)$. Setting $\delta=2$ it follows from [30, Corollary 2] that equation (14) can be solved with $u \in L_{2}^{2}\left(M_{V} ; \mathbb{R}\right)$. Put now $\delta^{\prime}:=-\frac{1}{2}+\varepsilon$ for any $\varepsilon>0$, and denote the interval $\left(\delta^{\prime}, \delta\right)$ by $I$. Notice that the only critical weights in $I$ are $\delta_{0}=\frac{3}{2}$ and $2-\delta_{0}=\frac{1}{2}$. It then follows from [30, Proposition 4 and Corollary 1] that up to perturbations in $L_{-\frac{1}{2}+\varepsilon}^{2}\left(M_{V} ; \mathbb{R}\right)$ and $L_{-\frac{3}{2}+\varepsilon^{\prime}, 2}^{2}\left(M_{V} ; \mathbb{R}\right)$ with some $\varepsilon^{\prime}>0$, $u$ is equal to a linear combination of solutions

$$
K_{0}^{0}(r)=r^{\nu_{0}^{+}}=1 \quad \text { and } \quad K_{-1}^{0}(r)=r^{\nu_{0}^{-}}=r^{-1}
$$

of the non-perturbed Laplacian. Notice that the corresponding spherical harmonic $Y_{0}^{0,0}=\frac{\sqrt{s}}{4 \pi}$ is constant and that the decay corresponding to $L_{-\frac{1}{2}+\varepsilon}^{2}\left(M_{V} ; \mathbb{R}\right)$ is $r^{\nu}$ with $\nu=-2+\varepsilon$, moreover the decay corresponding to $L_{-\frac{3}{2}+\varepsilon^{\prime}, 2}^{2}\left(M_{V} ; \mathbb{R}\right)$ is even stronger. Consequently (9) asymptotically also looks like

$$
f_{y, \lambda}(r)=1+\lambda r^{-1}+O\left(r^{-2+\varepsilon}\right)
$$

and the leading term is of course positive for $\lambda>0$. One can also immediately read off this from the explicit Green function of Page [31, Equation (18)].

By the aid of these observations we obtain 
Lemma 4.2. The smooth anti-self-dual connection $\nabla_{\tilde{A}_{y, \lambda}}$ with $y \in M_{V}$ and $\lambda \in(0,+\infty)$ is subject to both the weak holonomy condition with respect to the trivial flat connection $\nabla_{\Theta}$ and the rapid decay condition of Definition 2.1 consequently it has not only finite energy but is moreover admissible i.e., its gauge equivalence class gives rise to an admissible anti-instanton on $\Sigma^{+}$. The energy of this anti-instanton is of one unit.

Proof. We already have seen that the spin connection on $\Sigma^{+}$is gauge equivalent to the trivial flat connection $\nabla_{\Theta}$.

Consider the neck $W \subset M_{V}$ of the multi-Taub-NUT manifold and using the gauge (7) along $W$ write $\left.\nabla_{\tilde{A}_{y, \lambda}}\right|_{W}=\mathrm{d}+\tilde{A}_{y, \lambda}$ with $\tilde{A}_{y, \lambda}=a_{y, \lambda}$ as in (2) where $a_{y, \lambda}$ is the perturbation term involving (9). The asymptotic shape of (9) looks like (15) in light of our previous calculations. This ensures us that if $\lambda$ is finite and positive, $\left|\mathrm{d} \log f_{\lambda}\right|_{g_{V}}$ as well as $\left|\nabla_{\Theta}\left(\mathrm{d} \log f_{\lambda}\right)\right|_{g_{V}}$ are $O\left(r^{-2}\right)$ along $W$. Consequently, since

$$
\left|a_{y, \lambda}\right|=\sqrt{\frac{3}{2}}\left|\mathrm{~d} \log f_{y, \lambda}\right|_{g_{V}}
$$

in the gauge (7), we also obtain that both $\left|a_{y, \lambda}\right|$ and $\left|\mathrm{d}_{\Theta} a_{y, \lambda}\right|$ are $O\left(r^{-2}\right)$ along $W$ for all $y \in M_{V}$ and $0<\lambda<+\infty$. We see that the first condition in (5) is also satisfied. Inserting this and $F_{\Theta}=0$ into (4) we can see that our connection can be viewed as a rapidly decaying perturbation of the trivial connection hence satisfies both the weak holonomy condition with respect to the trivial flat connection as well as the rapid decay condition.

It follows $\left[13\right.$, Theorem 4.2] that in this situation the energy of the connection $\nabla_{\tilde{A}_{y, \lambda}}$ is integer hence it must be one because the harmonic function in (9) has only one singularity. $\diamond$

Remark. Before summarizing our findings we may raise the question: what about the higher overtones with $j>0$ in the harmonic expansion (13)? The radial function $r^{2}=|u|^{2}+|v|^{2}$ in $\mathbb{C}^{2}$ is invariant under $\mathbb{Z}_{s}$ consequently we can talk about lens spaces of radius $r$ centered at the origin of $\mathbb{C}^{2} / \mathbb{Z}_{s}$. Take such a lense space with its metric $g$ inherited from the round metric of $S^{3}$. By orthogonality the spherical harmonics obey for $j>0$ that

$$
\frac{1}{2} \int_{L(s,-1)}\left(Y_{j}^{k, l}+Y_{j}^{-k,-l}\right) \mathrm{d} V_{g}=\frac{1}{2 \mathbf{i}} \int_{L(s,-1)}\left(Y_{j}^{k, l}-Y_{j}^{-k,-l}\right) \mathrm{d} V_{g}=0
$$

hence these real functions must change sign somewhere along any lense space showing that in fact all individual terms in (13) with $j>0$ vanish on three dimensional subsets of $M_{V}^{*}$. Additionally we know from the asymptotic formulae for the radial functions $K_{-j-1}^{l}$ and $K_{j}^{l}$ that they behave very differently for small or large $r$ 's consequently the aforementioned three dimensional zero sets of the individual higher overtones cannot be removed by forming particular linear combinations of them. Consequently these harmonic functions represent "non-mild" singularities hence cannot be used to construct anti-instantons.

Let $\left.\nabla_{\Gamma}\right|_{W}$ be a smooth flat $\mathrm{SU}(2)^{+}$connection on $\left.\Sigma^{+}\right|_{W}$. Then following [13] if $C_{0}^{\infty}\left(M_{V}\right.$; End $\left.\Sigma^{+}\right)$ is the space of smooth, compactly supported endomorphisms of the positive chiral spin bundle then define the $L^{2}$ gauge group $\mathscr{G}_{\Sigma^{+}}$as the $L_{2, \Gamma}^{2}$ completion of the space

$$
\left\{\gamma-1 \in C_{0}^{\infty}\left(M_{V} ; \operatorname{End} \Sigma^{+}\right) \mid\|\gamma-1\|_{L_{2, \Gamma}^{2}\left(M_{V}\right)}<+\infty, \gamma \in C^{\infty}\left(M_{V} ; \operatorname{Aut} \Sigma^{+}\right) \text {a.e. }\right\}
$$

Let us denote by $\mathscr{M}(e, \Gamma)$ the framed moduli space consisting of pairs $\left(\left[\nabla_{A}\right], \Gamma\right)$, the $\mathscr{G}_{\Sigma^{+}}$gauge equivalence classes of smooth irreducible $\mathrm{SU}(2)^{+}$anti-self-dual connections on $\Sigma^{+}$of energy 
$e<+\infty$, decaying rapidly and obeying the weak holonomy condition with respect to $\left.\nabla_{\Gamma}\right|_{W}$ and a fixed gauge $\Gamma$ at infinity preserved by $\mathscr{G}_{\Sigma^{+}}$. Associated to this space one has the unframed moduli space $\widehat{\mathscr{M}}(e, \Gamma)$ consisting of the $\mathscr{G}_{\Sigma^{+}}$equivalence classes $\left[\nabla_{A}\right]$ only. In other words it is formed by dividing via the group

$$
\widehat{\mathscr{G}}_{\Sigma^{+}} \cong \mathscr{G}_{\Sigma^{+}} \times \mathrm{SU}(2)^{+}
$$

of gauge transformations tending to any fixed element $\gamma_{0} \in \mathrm{SU}(2)^{+}$(not to the identity only) at infinity.

In particular we can consider the spaces $\mathscr{M}(k, \Theta)$ and $\widehat{\mathscr{M}}(k, \Theta)$ corresponding to the trivial flat connection $\nabla_{\Theta}$; in this case the energy is an integer $k \in \mathbb{N}$, cf. [13, Theorem 4.2]. It was demostrated in [13, Theorem 3.2] that these spaces are smooth manifolds and [13, Theorem 4.2] ensures us that $\operatorname{dim} \mathscr{M}(k, \Theta)=8 k$ and therefore $\operatorname{dim} \widehat{\mathscr{M}}(k, \Theta)=8 k-3$.

Putting together our findings sofar as well as noting that all of our anti-instantons are irreducible [12, Theorem 5.1] we obtain the following

Theorem 4.1. Let $\left(M_{V}, g_{V}\right)$ be the multi-Taub-NUT space with $s>0$ NUTs equipped with an orientation coming from any of its complex strucures in the hyper-Kähler family.

Then there exists a 5 real parameter family of unframed, non-gauge equivalent, irreducible, smooth $\mathrm{SU}(2)^{+}$anti-instantons

$$
\left\{\left[\nabla_{\tilde{A}_{y, \lambda}}\right] \mid(y, \lambda) \in M_{V} \times(0,+\infty)\right\}
$$

on the positive chiral spinor bundle $\Sigma^{+}$which satisfy the weak holonomy condition with respect to the trivial flat connection and also satisfy the rapid decay condition. All these anti-instantons have unit energy and provide us with an open subset of (one connected component of) the unframed moduli space $\widehat{\mathscr{M}}(1, \Theta)$. $\diamond$

Theorem 4.1 can be regarded as a familiar "collar theorem" for the 1-anti-instanton moduli space over the multi-Taub-NUT space. The patient reader may write down these solutions explicitly by inserting the explicit Green functions [31, Equation (18)] of Page into (9) and then by the aid of the gauge (7) construct the corresponding connection 1-form via (2). However the quite complicated result is not informative.

Rather we turn attention to a puzzling feature of the description provided by Theorem 4.1. This is the limit $\lambda \rightarrow+\infty$. In this case (9) up to reparametrization remains meaningful and provides us with the positive minimal Green function $G(\cdot, y)$. It readily follows from the proofs of Lemmata 4.1 and 4.2 that the resulting anti-self-dual connection $\nabla_{\tilde{A}_{y,+\infty}}$ gives rise to an unframed anti-instanton $\left[\nabla_{\tilde{A}_{y,+\infty}}\right]$ on $\Sigma^{+}$obeying the weak holonomy condition with respect to $\nabla_{\Theta}$ and the rapid decay condition hence these solutions are expected to complete the moduli space.

Indeed, for a fixed $y \in M_{V}$ taking the limit $\lambda \rightarrow+\infty$ (especially in (10)) the proof of Lemma 4.1 continues to hold hence the corresponding anti-self-dual connection is smooth everywhere on $\Sigma^{+}$.

Additionally, in light of (15) the asymptotics of $G(\cdot, y)$ looks like $1 / r$ therefore the proof of Lemma 4.2 also can be repeated if we notice that this time $\left|a_{y}\right|$ and $\left|\mathrm{d}_{\Theta} a_{y}\right|$ are $O\left(r^{-1}\right)$ only. Hence $a_{y} \notin L_{\frac{1}{2}, 1, \Theta}^{2}\left(M_{V} ; \Lambda^{1} M_{V} \otimes\right.$ End $\left.\Sigma^{+}\right)$but it follows from a direct calculation that the corresponding curvature still belongs to the weighted Sobolev space: $F_{\tilde{A}_{y}} \in L_{\frac{1}{2}}^{2}\left(M_{V} ; \Lambda^{2} M_{V} \otimes \operatorname{End} \Sigma^{+}\right)$. We conclude that $\left[\nabla_{\tilde{A}_{y,+\infty}}\right]$ provides us with an admissible unframed anti-instanton with unit energy 
on the positive spin bundle; more precisely it decays rapidly and one can also check that the weak holonomy condition with respect to the same trivial flat connection $\nabla_{\Theta}$ is fulfilled. If $y \in M_{V}$ is not a NUT then the corresponding anti-instanton is irreducible otherwise reducible to $\mathrm{U}(1)$ and these Abelian solutions span the full $L^{2}$ cohomology of the multi-Taub-NUT geometry as it was stated in [12, Theorem 5.1].

Therefore if $y \in M_{V}$ is not a NUT then these limiting irreducible solutions are expected to give the completion of the collar $M_{V} \times(0,+\infty)$ to the full unframed moduli space in the unconcentrated or "centerless" regime - or at least one connected component of the moduli space emerges this way. Since it is natural to include the reducible solutions corresponding to the NUTs into $\widehat{\mathscr{M}}(1, \Theta)$ as well, for simplicity we shall continue to denote (one connected component of) this extended moduli space by $\widehat{\mathscr{M}}(1, \Theta)$ from now on and note that it is not a manifold anymore: usual conical singularities appear around the reducible points as we know from the general theory but will also see shortly.

We also conjecturially note that in our opinion not only a connected component but the whole moduli space of unit energy admissible anti-instantons with trivial holonomy arises this way.

Theorem 4.2. Consider the multi-Taub-NUT space $\left(M_{V}, g_{V}\right)$ with $s>0$ NUTs $p_{1}, \ldots, p_{s} \in M_{V}$, equipped with the natural orientation as above. Then for (one connected component of) the unframed moduli space of unit energy $\mathrm{SU}(2)^{+}$admissible anti-instantons decaying rapidly to the trivial flat connection on $\Sigma^{+}$we find

$$
\widehat{\mathscr{M}}(1, \Theta) \cong\left(M_{V} \times(0,+\infty]\right) / \sim
$$

where the equivalence relation $\sim$ means that $M_{V} \times\{+\infty\}$ is pinched into $\mathbb{R}^{3}$ by collapsing the $S^{1}$-isometry orbits of $\left(M_{V}, g_{V}\right)$.

Consequently there exists a singular fibration

$$
\Phi: \widehat{\mathscr{M}}(1, \Theta) \longrightarrow \mathbb{R}^{3}
$$

with generic fibers homeomorphic to the open 2-ball $B^{2}$ and as many as s singular fibers homeomorphic to the semi-open 1-ball $(0,+\infty]$. Therefore (one connected component of) the moduli space is contractible and in particular is orientable.

The images of the points $\left(p_{i},+\infty\right)$ in $\widehat{\mathscr{M}}(1, \Theta)$ with $i=1,2, \ldots, s$ represent reducible antiinstantons and $\widehat{\mathscr{M}}(1, \Theta)$ around these points looks like a cone over $\mathbb{C} P^{2}$ or $\overline{\mathbb{C P}}^{2}$ (depending on the orientation).

Proof. Athough we stated it already in Theorem 4.1 first of all we remark that from the expression (10) of the curvature one can immediately read off that both $y \in M_{V}$ and $\lambda \in(0,+\infty)$ are gauge-invariant parameters i.e., anti-instantons corresponding to different values of $(y, \lambda)$ cannot be gauge equivalent. Next, in Sect. 5 we will prove that if $y \in M_{V}$ and $\mathrm{e}^{\mathbf{i} \tau} y$ is its image by an element of the $S^{1}$ isometry group of $\left(M_{V}, g_{V}\right)$ then the corresponding two anti-instantons with $(y, \lambda=+\infty)$ and $\left(\mathrm{e}^{\mathbf{i} \tau} y, \lambda=+\infty\right)$ are actually gauge equivalent unit energy, rapidly decaying anti-instantons with trivial holonomy at infinity.

In addition to these we know from [13, Theorem 3.2] that away from the reducible points the moduli space is a finite dimensional, possibly non-connected manifold without boundary.

Consequently we are forced to look at our moduli space as follows. Fix a point $y \in M_{V}$ different from any NUT and consider the $S^{1}$ isometry orbit through $y$ : it is parameterized by the cyclic coordinate $\tau \in[0,2 \pi)$ of the local coordinate system on $\left(M_{V}, g_{V}\right)$ introduced in Sect. 
3 ; as well as take the concentration parameter $\lambda \in(0,+\infty)$. Then by Theorem 4.1, associated to any $y \in M_{V}$, we obtain a subset of the moduli space paremeterized by $(\tau, \lambda)$. We regard this as a punctured open $B^{2}$ such that the points with $\lambda=0$ are supposed to represent the boundary while $\lambda=+\infty$ is the center of this $(\tau, \lambda)$-ball. In this picture we interpret the existence of smooth solutions with $\lambda=+\infty$ as filling in the punctured $(\tau, \lambda)$-ball over $y \in M_{V}$ with the anti-instanton $\left[\nabla_{\tilde{A}_{y,+\infty}}\right]$ corresponding to the limit $\lambda \rightarrow+\infty$. In other words, this anti-instanton represents the center of the unpunctured $(\tau, \lambda)$-ball that consequently will be homeomorphic to an open $B^{2}$ over any $y \in M_{V}$ different from the NUTs.

Since the NUTs $p_{1}, \ldots, p_{s} \in M_{V}$ are the only fixed points of the isometry group action on $\left(M_{V}, g_{V}\right)$ it follows that over these points the moduli space is parameterized by $\lambda \in(0,+\infty]$ only hence is a semi-open $B^{1}$. In this case the "center" is the endpoint $\lambda=+\infty$.

Therefore completing our collar $M_{V} \times(0,+\infty)$ with these limiting solutions, the moduli space takes the shape as in (17) where the equivalence relation $\sim$ means that $M_{V} \times\{+\infty\}$ is collapsed into $\mathbb{R}^{3}$ by shrinking all the isometry orbits of $\left(M_{V}, g_{V}\right)$ into single points respectively and admits a singular fibration (18) whose generic fiber is an open $B^{2}$ and with $s$ pieces of singular fibers homeomorphic to a semi-open $B^{1}$. In particular $\widehat{\mathscr{M}}(1, \Theta)$ is contractible hence orientable.

We already know that the only reducible solutions are the images $r_{i} \in \widehat{\mathscr{M}}(1, \Theta)$ of the points $\left(p_{i},+\infty\right)$ with $i=1,2, \ldots, s$. We will study the structure of the moduli space around these points. Take a NUT $p_{i} \in M_{V}$ and consider its neighbourhood $U\left(r_{i}\right) \subset \widehat{\mathscr{M}}(1, \Theta)$. We will construct a decomposition $\partial \bar{U}\left(r_{i}\right)=X_{1} \cup X_{2}$ of its boundary as follows. It is clear that if $y$ is not a NUT and the image of $(y,+\infty)$ in $\widehat{\mathscr{M}}(1, \Theta)$ is $m$ then $U(m) \cong B^{5}$, an open 5-ball. Let us decompose its closure as $\bar{U}(m) \cong \bar{B}^{3} \times \bar{B}^{2}$ where $B^{2}$ is the aforementioned $(\tau, \lambda)$-disk and $B^{3}$ is a ball around the image of $y$ in $\mathbb{R}^{3}$. Then $\partial \bar{U}(m) \cong\left(S^{2} \times \bar{B}^{2}\right) \cup\left(\bar{B}^{3} \times S^{1}\right)$ which is of course an $S^{4}$ glued together from $S^{2} \times \bar{B}^{2}$ and $\bar{B}^{3} \times S^{1}$ along their common boundary $S^{2} \times S^{1}$. Now let us move $y$ toward a particular NUT $p_{i}$; then one of the $\bar{B}^{2}$ s in the $S^{2} \times \bar{B}^{2}$ component of the above decomposition, namely that one which is moved exactly into the position of the NUT, gets shrink to a $\bar{B}^{1}$ in a manner such that meanwhile its radius (measured by $\lambda$ ) is kept constant, its circumference (measured by $\tau$ ) gets vanish. ${ }^{3}$ Put

$$
X_{1} \cong S^{2} \times \bar{B}^{2} / \sim_{1}
$$

corresponding to this collapsed space. We obviously find for the singular cohomology that

$$
H^{j}\left(X_{1} ; \mathbb{Z}\right) \cong \begin{cases}\mathbb{Z} & \text { if } j=0,2 \\ 0 & \text { otherwise }\end{cases}
$$

Since this movement also collapses an $S^{1}$, the boundary in the other component $\bar{B}^{3} \times S^{1}$ of the previously collapsed $\bar{B}^{2}$, we also get a corresponding collapsed space

$$
X_{2} \cong \bar{B}^{3} \times S^{1} / \sim_{2} .
$$

Since the generator of $\pi_{1}\left(\bar{B}^{3} \times S^{1}\right) \cong \mathbb{Z}$ is just killed out in $X_{2}$, we also obtain

$$
H^{j}\left(X_{2} ; \mathbb{Z}\right) \cong \begin{cases}\mathbb{Z} & \text { if } j=0 \\ 0 & \text { otherwise }\end{cases}
$$

\footnotetext{
${ }^{3}$ This way of collapsing $\bar{B}^{2}$ into $\bar{B}^{1}$ looks like closing an umbrella.
} 
It is also clear that $X_{1} \cap X_{2}$ is nothing else than a 3-sphere with exactly two antipodal points pinched consequently

$$
H^{j}\left(X_{1} \cap X_{2} ; \mathbb{Z}\right) \cong \begin{cases}\mathbb{Z} & \text { if } j=0,3 \\ 0 & \text { otherwise }\end{cases}
$$

Recalling the Mayer-Vietoris sequence of the decomposition $\partial \bar{U}\left(r_{i}\right)=X_{1} \cup X_{2}$ which looks like

$$
\cdots \rightarrow H^{j}\left(\partial \bar{U}\left(r_{i}\right) ; \mathbb{Z}\right) \rightarrow H^{j}\left(X_{1} ; \mathbb{Z}\right) \oplus H^{j}\left(X_{2} ; \mathbb{Z}\right) \rightarrow H^{j}\left(X_{1} \cap X_{2} ; \mathbb{Z}\right) \rightarrow H^{j+1}\left(\partial \bar{U}\left(r_{i}\right) ; \mathbb{Z}\right) \rightarrow \ldots
$$

we end up with

$$
H^{j}\left(\partial \bar{U}\left(r_{i}\right) ; \mathbb{Z}\right) \cong \begin{cases}\mathbb{Z} & \text { if } j=0,2,4 \\ 0 & \text { otherwise. }\end{cases}
$$

Moreover we know that $\partial \bar{U}\left(r_{i}\right)$ is simply connected and is smooth. However from all of these we obtain via Freedman's classification [14] that $\partial \bar{U}\left(r_{i}\right) \cong \mathbb{C} P^{2}$ or $\overline{\mathbb{C} P}^{2}$ depending on the orientation put onto $\widehat{\mathscr{M}}(1, \Theta)$. Therefore the unframed moduli space about a reducible point looks like a cone over one of these spaces as stated. $\diamond$

We make a comment on the higher-energy moduli spaces including anti-instantons with trivial holonomy in infinity. It is obvious that instead of the functions (9) we can use harmonic functions with a finite number of isolated singularities yielding

Theorem 4.3. The moduli spaces $\mathscr{M}(k, \Theta)$ hence $\widehat{\mathscr{M}}(k, \Theta)$ are non-empty for all $k \in \mathbb{N}$.

For sake of completeness without proof we exhibit here the degenerate $s=0$ case as well i.e., the flat $\mathbb{R}^{3} \times S^{1}$ whose associated instantons also referred to as calorons. This space is flat hence conformal rescaling works. Using coordinates $\left(x^{1}, x^{2}, x^{3}, \tau\right)=(x, \tau)$ on $\mathbb{R}^{3} \times S^{1}$ we have an obvious five paramerer family of positive harmonic functions (9) with Green functions in a rather explicit form [31]

$$
G\left(\left(x, \tau_{x}\right),\left(y, \tau_{y}\right)\right)=\frac{1}{16 \pi^{2}|x-y|} \frac{\tanh |x-y|}{1-\frac{\cos \left(\tau_{x}-\tau_{y}\right)}{\cosh |x-y|}}=\frac{1}{4 \pi^{2}} \sum_{k=-\infty}^{+\infty} \frac{1}{|x-y|^{2}+\left(\tau_{x}-\tau_{y}+2 \pi k\right)^{2}} .
$$

Making use of [13, Theorem 2.3] to describe the holonomy issue in this situation as well as [13, Theorem 4.1] which provides us with the dimension moreover taking into account that there are no reducible $\mathrm{SU}(2)^{ \pm}$instantons on this space we state

Theorem 4.4. Take the flat $\mathbb{R}^{3} \times S^{1}$ with an arbitrary fixed orientation. Then any non-trivial $\mathrm{SU}(2)^{ \pm}$instanton on $\Sigma^{ \pm}$is irreducible moreover the rapidly decaying ones obey the weak holonomy condition with respect to some flat connection $\nabla_{\Gamma}$ on the neck and their energies are always nonnegative integers $k \in \mathbb{N}$. The corresponding framed moduli spaces, if not empty, have dimensions $\operatorname{dim} \mathscr{M}(k, \Gamma)=8 k$ while $\operatorname{dim} \widehat{\mathscr{M}}(k, \Gamma)=8 k-3$ for the unframed ones.

Assume the asymptotic flat connection is the trivial one $\nabla_{\Theta}$ and let $\mathscr{M}(1, \Theta)$ be the corresponding framed moduli space. Then for (one connected component of) the unframed space we find

$$
\widehat{\mathscr{M}}(1, \Theta) \cong\left(\mathbb{R}^{3} \times S^{1} \times(0,+\infty]\right) / \sim \cong \mathbb{R}^{3} \times B^{2}
$$

moreover the higher energy framed moduli spaces $\mathscr{M}(k, \Theta)$ hence $\widehat{\mathscr{M}}(k, \Theta)$ are also non-empty with $k \in \mathbb{N}$ arbitrary. $\diamond$ 


\section{Completion of the moduli space}

In this closing section our aim is to prove that conformal rescaling gives rise to at least one connected component of unit energy, rapidly decaying anti-instantons obeying the weak holonomy condition with respect to the trivial flat connection (however we note again that in our opinion this moduli space is connected).

Our starting point is the construction of the twistor space of the multi-Taub-NUT geometry by the aid of Hitchin $[6,22,24]$ and also a certain compactification of it. Then following Atiyah [2] this enables us to identify the harmonic functions used so far with elements of certain Ext groups on the compactified twistor space. These Ext groups also provide us with twisted holomorphic vector bundles over the original non-compact twistor space and the corresponding untwisted ones represent our anti-instantons in a new form in the spirit of the Atiyah-Ward correspondence.

To get these twisted vector bundles more explicitly we investigate the real structure of the twistor space leading to the multi-Taub-NUT geometry and also construct the corresponding real twistor lines; our twisted vector bundles then, referring to Serre's method, can be constructed via sections vanishing along these real lines. The transition functions of these twisted bundles corresponding to pure Green functions, i.e., in the $\lambda \rightarrow+\infty$ limit can be constructed rather explicitly. We will find that the twisted bundles corresponding to $G(\cdot, y)$ and $G\left(\cdot, \mathrm{e}^{\mathbf{i} \tau} y\right)$ are actually isomorphic hence the corresponding anti-instantons must be gauge equivalent verifying our picture on the moduli space presented in Theorem 4.2.

To begin with, we claim that there exist nice compactifications of the complex manifolds underlying a given a multi-Taub-NUT space.

Lemma 5.1. Consider any multi-Taub-NUT space $\left(M_{V}, g_{V}\right)$ with s NUTs and let $X$ denote the smooth complex surface constructed in Lemma 3.1 given by picking one generic complex structure in the hyper-Kähler family. Also let $\widetilde{X}$ be the smooth complex surface belonging to any exceptional complex structure, as constructed in Lemma 3.1.

Then both $X$ and $\widetilde{X}$ admit compactifications $\bar{X}$ and $\overline{\widetilde{X}}$ respectively which are smooth compact rational surfaces. These spaces arise by attaching $s+3$ lines to the finite parts in a suitable way.

Proof. Fix a generic configuration of $s$ points $q_{1}, \ldots, q_{s} \in \mathbb{R}^{3}$ and consider the corresponding multi-Taub-NUT space $\left(M_{V}, g_{V}\right)$. Pick a direction $e_{1}$ in $\mathbb{R}^{3}$ not parallel with any straight line segment $\ell_{i j}$ connecting $q_{i}$ and $q_{j}$ and put the corresponding (generic) integrable complex structure $J_{e_{1}}$ onto $M_{V}$ from the hyper-Kähler family. We know from Lemma 3.1 that $\left(M_{V}, J_{e_{1}}\right)$ is biholomorphic to the algebraic surface $X \subset \mathbb{C}^{3}$ given by $(8)$ and there are $s(s-1)$ exceptional directions to choose for $e_{1}$ so that the corresponding complex manifolds are not biholomorphic to $X$ but rather to its blowup denoted by $\widetilde{X}$ in agreement with the notations of Lemma 3.1.

First consider the case of $X$. Introducing homogeneous coordinates $([x: u],[y: v],[z: w]) \in$ $\mathbb{C} P_{x}^{1} \times \mathbb{C} P_{y}^{1} \times \mathbb{C} P_{z}^{1}$ equation (8) can be made homogeneous of degrees $(1,1, s)$ respectively as follows:

$$
x y w^{s}-u v \prod_{i=1}^{s}\left(z-w p_{i}\right)=0 .
$$

Let us denote the resulting compact complex surface in $\mathbb{C} P_{x}^{1} \times \mathbb{C} P_{y}^{1} \times \mathbb{C} P_{z}^{1}$ by $\bar{X}^{*}$. We claim that $\bar{X}^{*}$ is smooth except one point. Indeed, let $0_{x}:=[0: 1]$ and $\infty_{x}:=[1: 0]$ denote the origin and the infinity of $\mathbb{C} P_{x}^{1}$ respectively and in the same fashion introduce the notation $0_{y}, \infty_{y} \in \mathbb{C} P_{y}^{1}$ 
and $0_{z}, \infty_{z} \in \mathbb{C} P_{z}^{1}$. Then in the compactification $\mathbb{C}^{3} \subset \mathbb{C} P_{x}^{1} \times \mathbb{C} P_{y}^{1} \times \mathbb{C} P_{z}^{1}$ infinity is represented by the bouquet

$$
\infty:=\left(\left\{\infty_{x}\right\} \times \mathbb{C} P_{y}^{1} \times \mathbb{C} P_{z}^{1}\right) \vee\left(\mathbb{C} P_{x}^{1} \times\left\{\infty_{y}\right\} \times \mathbb{C} P_{y}^{1}\right) \vee\left(\mathbb{C} P_{x}^{1} \times \mathbb{C} P_{y}^{1} \times\left\{\infty_{z}\right\}\right)
$$

and one obtains that $\bar{X}^{*} \cap \infty$ consists of four lines, that is $\bar{X}^{*}=X \sqcup\left(\ell_{1} \cup \ell_{2} \cup \ell_{3} \cup \ell_{4}\right)$ with

$$
\begin{array}{ll}
\ell_{1}:=\left\{\infty_{x}\right\} \times\left\{0_{y}\right\} \times \mathbb{C} P_{z}^{1}, \quad \ell_{2}:=\left\{\infty_{x}\right\} \times \mathbb{C} P_{y}^{1} \times\left\{\infty_{z}\right\}, \\
\ell_{3}:=\mathbb{C} P_{x}^{1} \times\left\{\infty_{y}\right\} \times\left\{\infty_{z}\right\}, \quad \ell_{4}:=\left\{0_{x}\right\} \times\left\{\infty_{y}\right\} \times \mathbb{C} P_{z}^{1} .
\end{array}
$$

These lines are not disjoint, they intersect each other as follows:

$$
\ell_{1} \cap \ell_{2}=\left(\infty_{x}, 0_{y}, \infty_{z}\right), \quad \ell_{2} \cap \ell_{3}=\left(\infty_{x}, \infty_{y}, \infty_{z}\right), \quad \ell_{3} \cap \ell_{4}=\left(0_{x}, \infty_{y}, \infty_{z}\right) .
$$

By calculating the gradient of the left hand side in (19) we see that the only point where $\bar{X}^{*}$ is singular is the intersection $\ell_{2} \cap \ell_{3}$ where $\bar{X}^{*}$ locally looks like

$$
u v-\prod_{i=1}^{s} w\left(1-w p_{i}\right)^{-1}=0
$$

i.e., it possesses a classical $A_{s-1}$ quotient singularity.

Carrying out the same procedure for the exceptional singular surface $X^{*} \subset \mathbb{C}^{3}$ with the notations of Lemma 3.1, we obtain a similar compactification ${\overline{X^{*}}}^{*}$ in $\mathbb{C} P_{x}^{1} \times \mathbb{C} P_{y}^{1} \times \mathbb{C} P_{z}^{1}$. The only difference is that in addition to the $A_{s-1}$ singularity at infinity as above, ${\overline{X^{*}}}^{*}$ possess more rational double points in the finite part in each point where $\left[p_{i}: 1\right]=\left[p_{j}: 1\right]$.

We proceed further and desingularize these singular compactifications. In light of Lemma 3.1 we simply do this by replacing them with their non-singular blowups. Again consider first the generic case $\bar{X}^{*}$ and let us denote the resulting smooth space by $\bar{X}$. Clearly, in this case we have to just remove the $A_{s-1}$-singularity at infinity from $\bar{X}^{*}$ therefore we have to attach $4+s-1=s+3$ lines in an approriate way:

$$
\bar{X}=X \sqcup\left(\ell_{1} \cup \ell_{2} \cup \ell_{1}^{\prime} \cup \cdots \cup \ell_{s-1}^{\prime} \cup \ell_{3} \cup \ell_{4}\right)
$$

to obtain $\bar{X}$. Denoting by $\overline{\widetilde{X}}$ the desingularization of ${\overline{X^{*}}}^{*}$, it stems exactly the same way from the smooth $\tilde{X}$, the desingularization of $X^{*} \subset \mathbb{C}^{3}$.

Finally we prove that both of these smooth compactifications are rational surfaces. Indeed, consider first general case when $\bar{X}^{*}$ is smooth except at infinity. Then $\bar{X}^{*}$ is given by equation (19) for distinct values of $p_{j}$ 's. Consider the map

$$
\bar{X}^{*} \longrightarrow \mathbb{C} P_{x}^{1} \times \mathbb{C} P_{z}^{1}, \quad([x: u],[y: v],[z: w]) \longmapsto([x: u],[z: w]) .
$$

Over a given $([x: u],[z: w]) \in \mathbb{C} P_{x}^{1} \times \mathbb{C} P_{z}^{1}$ there exists a unique $[y: v] \in \mathbb{C} P_{y}^{1}$ such that $([x: u],[y: v],[z: w]) \in \bar{X}^{*}$, except in the case $x w^{s}=u \prod\left(z-w p_{j}\right)=0$ when any $[y: v]$ is a solution. It readily follows that these exceptional points are precisely

$$
\left\{\left(0_{x},\left[p_{1}: 1\right]\right), \ldots,\left(0_{x},\left[p_{s}: 1\right]\right),\left(\infty_{x}, \infty_{z}\right)\right\} \in \mathbb{C} P_{x}^{1} \times \mathbb{C} P_{z}^{1} .
$$

Furthermore, all the points of $\bar{X}^{*}$ are non-singular except the isolated hence codimension 2 surface singularity of type $A_{s-1}$ at $\left(\infty_{x}, \infty_{y}, \infty_{z}\right) \in \mathbb{C} P_{x}^{1} \times \mathbb{C} P_{y}^{1} \times \mathbb{C} P_{z}^{1}$ which is mapped onto 
$\left(\infty_{x}, \infty_{z}\right)$ in $\mathbb{C} P_{x}^{1} \times \mathbb{C} P_{z}^{1}$. It follows that $\bar{X}^{*}$ is isomorphic to the blowup of $\mathbb{C} P_{x}^{1} \times \mathbb{C} P_{z}^{1}$ in the points (22) outside of a codimension 2 subset. The same argument works for the special case of $\bar{X}^{*}$ possessing a further rational double point, if we add this singular point to the codimension 2 subset above. Therefore both $\bar{X}^{*}$ and $\bar{X}^{*}$ are rational. Since both $\bar{X}$ and $\overline{\widetilde{X}}$ arise by blowing further up $\bar{X}^{*}$ and $\bar{X}^{*}$ respectively in finitely many points, we conclude that the non-singular compact spaces $\bar{X}$ and $\overline{\widetilde{X}}$ are also rational. $\diamond$

Remark. It is worth comparing the complex compactification (21) with the simple smooth real compactification of $M_{V}$ in $[11,13]$ motivated by $L^{2}$-cohomology theory of ALF spaces [19].

We proceed further and smoothly compactify the twistor space of a multi-Taub-NUT space in two steps.

Lemma 5.2. The first approximation of the twistor space (what we denote here by) $Z$ of any multi-Taub-NUT space $\left(M_{V}, g_{V}\right)$ with s NUTs admits a smooth complex compactification (what we denote here by) $\bar{Z}$. This compactification arises by adding finitely many various Hirzebruch surfaces to this $Z$ in a suitable way.

Proof. First we construct a sort of approximation of the twistor space of $\left(M_{V}, g_{V}\right)$ and then modify it into the true twistor space in the next lemma.

Being $\left(M_{V}, g_{V}\right)$ simply connected with vanishing Ricci curvature, its true twistor space admits a holomorphic fibration over $\mathbb{C} P^{1}$ consequently we would like to regard equation (19) as a set of equations parameterized by a projective line. Therefore we proceed as follows (cf. [6, pp. 393-395] and [22]). Consider the holomorphic fiber bundle

$$
\bar{\pi}: P\left(H^{k} \oplus H^{0}\right) \oplus P\left(H^{l} \oplus H^{0}\right) \oplus P\left(H^{m} \oplus H^{0}\right) \longrightarrow \mathbb{C} P^{1}
$$

whose fibers are $F_{x} \times F_{y} \times F_{y}$ with $F_{i} \cong \mathbb{C} P_{i}^{1}(i=x, y, z)$ as before. Take the subbundle $\bar{\pi}_{x}: P\left(H^{k} \oplus H^{0}\right) \rightarrow \mathbb{C} P^{1}$ with $F_{x} \cong \mathbb{C} P_{x}^{1}$. Referring to the isomorphism

$$
P\left(H^{0} \oplus H^{-k}\right) \stackrel{\otimes H^{k}}{\longrightarrow} P\left(H^{k} \oplus H^{0}\right)
$$

take the canonical section $\infty_{x}:=(1,0)$ of $P\left(H^{0} \oplus H^{-k}\right)$ and regard its image as a divisor which we also denote by $\infty_{x}$; one then has an associated line bundle $L_{x}:=\left[\infty_{x}\right]$ and corresponding sheaf $\mathscr{O}_{\text {rel }, x}(1)$ on $P\left(H^{0} \oplus H^{-k}\right)$. This line bundle has a canonical section $u \in \mathscr{O}_{\text {rel }, x}(1)$ satisfying $(u)=\infty_{x}$. We also have a canonical section $0_{x}:=(0,1)$ of $P\left(H^{k} \oplus H^{0}\right)$ which gives rise to the bundle $L_{x} \otimes \bar{\pi}_{x}^{*} H^{k}$ and a canonical section $x$ which is an element of $\mathscr{O}_{\text {rel, } x}(1) \otimes \mathscr{O}_{P\left(H^{k} \oplus H^{0}\right)}(k)$ where $\mathscr{O}_{P\left(H^{k} \oplus H^{0}\right)}(k)$ is the associated sheaf of $\pi_{x}^{*} H^{k}$. Take the fibration

$$
\bar{\pi}_{y, z}: P\left(H^{k} \oplus H^{0}\right) \oplus P\left(H^{l} \oplus H^{0}\right) \oplus P\left(H^{m} \oplus H^{0}\right) \longrightarrow P\left(H^{k} \oplus H^{0}\right)
$$

whose fibers are $F_{y} \times F_{z}$. Pulling back $x, u$ we therefore obtain elements

$$
\bar{\pi}_{y, z}^{*} x \in \bar{\pi}_{y, z}^{*} \mathscr{O}_{\mathrm{rel}, x}(1) \otimes \bar{\pi}_{y, z}^{*} \mathscr{O}_{P\left(H^{k} \oplus H^{0}\right)}(k), \quad \bar{\pi}_{y, z}^{*} u \in \bar{\pi}_{y, z}^{*} \mathscr{O}_{\mathrm{rel}, x}(1) .
$$

In the same fashion we construct

$$
\bar{\pi}_{x, z}^{*} y \in \bar{\pi}_{x, z}^{*} \mathscr{O}_{\mathrm{rel}, y}(1) \otimes \bar{\pi}_{x, z}^{*} \mathscr{O}_{P\left(H^{l} \oplus H^{0}\right)}(l), \quad \bar{\pi}_{x, z}^{*} v \in \bar{\pi}_{x, z}^{*} \mathscr{O}_{\mathrm{rel}, y}(1)
$$


and finally

$$
\bar{\pi}_{x, y}^{*} z \in \bar{\pi}_{x, y}^{*} \mathscr{O}_{\mathrm{rel}, z}(1) \otimes \bar{\pi}_{x, y}^{*} \mathscr{O}_{P\left(H^{m} \oplus H^{0}\right)}(m), \quad \bar{\pi}_{x, y}^{*} w \in \bar{\pi}_{x, y}^{*} \mathscr{O}_{\mathrm{rel}, z}(1) .
$$

For simplicity we shall denote them as $x, u, y, v, z, w$ respectively and observe that they provide us with coordinates on the total space of the fibration (23).

The situation gets simplified somewhat by virtue of the following observation. It is not difficult to check that the bundle $\bar{\pi}_{y, z}^{*}\left(\bar{\pi}_{x}^{*} H^{k}\right)$ on the total space of (23) depends only on $k$ and not on the way we pulled it back from $\mathbb{C} P^{1}$. Consequently

$$
\bar{\pi}_{y, z}^{*}\left(\bar{\pi}_{x}^{*} H^{k}\right) \cong \bar{\pi}_{x, y}^{*}\left(\bar{\pi}_{z}^{*} H^{k}\right) \cong \bar{\pi}_{x, z}^{*}\left(\bar{\pi}_{y}^{*} H^{k}\right) \cong \bar{\pi}^{*} H^{k}
$$

and the same is true for the other bundles $H^{l}$ and $H^{m}$. Write

$$
W:=P\left(H^{k} \oplus H^{0}\right) \oplus P\left(H^{l} \oplus H^{0}\right) \oplus P\left(H^{m} \oplus H^{0}\right)
$$

for the total space of (23) and put

$$
L^{a_{0}, a_{1}, a_{2}, a_{3}}:=\bar{\pi}^{*} H^{a_{0}} \otimes \bar{\pi}_{y, z}^{*} L_{x}^{a_{1}} \otimes \bar{\pi}_{x, z}^{*} L_{y}^{a_{2}} \otimes \bar{\pi}_{x, y}^{*} L_{z}^{a_{3}}
$$

with $a_{i} \in \mathbb{Z}$; we will also use the convenient notation for the corresponding sheaf:

$$
\mathscr{O}_{W}\left(a_{0}, a_{1}, a_{2}, a_{3}\right):=\mathscr{O}_{W}\left(a_{0}\right) \otimes \bar{\pi}_{y, z}^{*} \mathscr{O}_{\mathrm{rel}, x}\left(a_{1}\right) \otimes \bar{\pi}_{x, z}^{*} \mathscr{O}_{\mathrm{rel}, y}\left(a_{2}\right) \otimes \bar{\pi}_{x, y}^{*} \mathscr{O}_{\mathrm{rel}, z}\left(a_{3}\right)
$$

(therefore $\left.\mathscr{O}_{W}\left(a_{0}\right)=\mathscr{O}_{W}\left(a_{0}, 0,0,0\right)\right)$. Then $x \in \mathscr{O}_{W}(k, 1,0,0)$ and $u \in \mathscr{O}_{W}(0,1,0,0)$, etc. and if we regard $p_{i} \in \mathscr{O}_{W}(m, 0,0,0)$ we obtain

$$
x y w^{s} \in \mathscr{O}_{W}(k+l, 1,1, s) \quad \text { and } \quad u v \prod_{i=1}^{s}\left(z-w p_{i}\right) \in \mathscr{O}_{W}(s m, 1,1, s)
$$

consequently setting $k+l=s m$ we end up with a well-defined map $\bar{P}: W \longrightarrow L^{s m, 1,1, s}$ where formally $\bar{P}$ is given by the left hand side of (19) and a singular model for the first approximation of the compactified twistor space $\bar{Z}^{*} \subset W$ is provided by the hypersurface $\bar{P}=0$ hence restricting (23) it admits a holomorphic fibration $\bar{\pi}: \bar{Z}^{*} \longrightarrow \mathbb{C} P^{1}$.

To simplify the notation of Lemma 5.1 from now on we shall denote the fibers $\bar{\pi}^{-1}([a: b])$ of this fibration as $\bar{X}_{[a: b]}^{*}$ for all $[a: b] \in \mathbb{C} P^{1}$. Note that $\bar{Z}^{*}$ has an obvious singularity along the line $\ell$ which is the section passing through the singular points $\left(\infty_{x}, \infty_{y}, \infty_{z}\right)_{[a: b]} \in \bar{X}_{[a: b]}^{*}$ for an $[a: b] \in \mathbb{C} P^{1}$. However $\bar{Z}^{*}$ has more singularities, namely the points $\left(0,0, p_{i}([a: b])\right)$ where exactly two roots coincide: $p_{i}([a: b])=p_{j}([a: b])$. (We may assume that the configuration is generic hence no more than two roots coincide at the same time.) Hence these points represent rational double points in $\bar{Z}^{*}$. Therefore the number of such bad points is $m \cdot\left(\begin{array}{l}s \\ 2\end{array}\right)=m \cdot \frac{1}{2} s(s-1)$ since $p_{i} \in \mathscr{O}_{W}(m, 0,0,0)$. The geometric origin of these bad points is clear: they just correspond to the special complex structures analyzed in Lemma 3.1 under which $\left(M_{V}, J_{e_{1}}\right)$ is not biholomorphic to (8). Since the number of these complex structures is $s(s-1)$ as we have seen, we have to set $m=2$ yielding $k+l=2 s$ and since $x$ and $y$ play symmetric roles, we take $k=l=s$ in (23).

We desingularize $\bar{Z}^{*}$ to obtain a smooth compact complex 3-manifold $\bar{Z}$ whose finite part $Z \subset \bar{Z}$ provides us with a non-singular model for the approximated twistor space of $\left(M_{V}, g_{V}\right)$. In light of Lemmata 3.1 and 5.1 we simply do this by replacing the fibers $\bar{X}_{[a: b]}^{*}$ of $\bar{\pi}: \bar{Z}^{*} \longrightarrow \mathbb{C} P^{1}$ with their non-singular blowups for all $[a: b] \in \mathbb{C} P^{1}$. Let us denote the resulting smooth fiber 
by $\bar{X}_{[a: b]}$. Since these fibers are diffeomorphic to each other via Lemma 3.1 we end up with a smooth holomorphic fibration; we denote it as $\bar{\pi}: \bar{Z} \longrightarrow \mathbb{C} P^{1}$.

Finally, referring to (21) we conclude that the compactified smooth $\bar{Z}$ arises by adding $s+3$ Hirzebruch surfaces to the smooth twistor space $Z$ of $\left(M_{V}, g_{V}\right)$ as follows:

$$
\bar{Z}=Z \sqcup\left(S_{1} \cup S_{2} \cup S_{1}^{\prime} \cup \cdots \cup S_{s-1}^{\prime} \cup S_{3} \cup S_{4}\right) .
$$

Two of them, $S_{1}$ and $S_{4}$ stemming from the lines $\ell_{1}$ and $\ell_{4}$ in $\bar{X}^{*}$, are biholomorphic to $P\left(H^{2} \oplus H^{0}\right)$ while $S_{2}$ and $S_{3}$ given by $\ell_{2}$ and $\ell_{3}$ are biholomorphic to $P\left(H^{s} \oplus H^{0}\right)$. To determine the type of the exceptional ones, notice that the local resolution of the singularity (20) is given by defining the new variables $u_{s}:=w^{-s} u, v_{s}:=v, w_{s}:=w$, whence the equation of the proper transform becomes

$$
u_{s} v_{s}-\prod_{i=1}^{s}\left(1-w_{s} p_{i}\right)^{-1}=0
$$

This means that we add a family of projective lines parametrized by $\mathbb{C} P^{1}$ with sections $w_{s}$. As $w_{s}$ is untwisted in the base direction, this implies that the Hirzebruch surfaces $S_{1}^{\prime}, \ldots, S_{s-1}^{\prime}$ we add through this process are trivial $\mathbb{C} P^{1} \times \mathbb{C} P^{1}$ 's. $\diamond$

Remark. The reader may recognize that this "first approximation" of the twistor space of the multi-Taub-NUT space is nothing else than the twistor space of the corresponding ALE GibbonsHawking space [22].

Lemma 5.3. The true twistor space $Z$ of any multi-Taub-NUT space $\left(M_{V}, g_{V}\right)$ with $s$ NUTs also admits a smooth complex compactification $\bar{Z}$. It also arises by adding $s+3$ Hirzebruch surfaces to $Z$ in a suitable way.

Proof. The idea is to twist (cf. [6, pp. 393-395] and [24]) the natural algebraic model constructed in Lemma 5.2 with a section of a certain line bundle $L^{c}$ which lives on the total space of the line bundle $\pi_{z}: H^{2} \rightarrow \mathbb{C} P^{1}$ where this base is the same as that of (23).

For the definition of $L^{c}$, let this $\mathbb{C} P^{1}$ be covered by the affine open sets $U_{a}$ and $U_{b}$ with coordinates $[a: b]$ satisfying $a \neq 0$ and $b \neq 0$ respectively and write $U_{a}^{\prime}=\pi_{z}^{-1}\left(U_{a}\right)$ and $U_{b}^{\prime}=$ $\pi_{z}^{-1}\left(U_{b}\right)$ for the open subsets of $H^{2}$ covering the affine subsets of $\mathbb{C} P^{1}$ moreover denote by $z$ any point in the total space of $H^{2}$. Therefore we obtain coordinate systems $\left(\left[1: \frac{b}{a}\right], z\right)$ and $\left(\left[\frac{a}{b}: 1\right], z\right)$ on $U_{a}^{\prime}$ and $U_{b}^{\prime}$ respectively. Define the holomorphic line bundle $L^{c}$ with a complex parameter $c$ by the transition function

$$
g: U_{a}^{\prime} \cap U_{b}^{\prime} \longrightarrow \mathbb{C}^{*}, \quad g(z,[a: b]):=\mathrm{e}^{-c z \frac{b}{a}} .
$$

It is clear that $L^{0}$ is just the trivial bundle over $H^{2}$ and $L^{-c}$ is canonically isomorphic to $\left(L^{c}\right)^{*}$.

Next we construct a section of $L^{c}$. For this we exploit a nice geometric description of $H^{2} \cong$ $T \mathbb{C} P^{1}$ by identifying it with the space of oriented affine lines in $\mathbb{R}^{3}$ (with a fixed origin) as follows. If $\ell \subset \mathbb{R}^{3}$ is an oriented affine line and $\ell(t) \in \ell$ is its point, $t \in \mathbb{R}$, then clearly exist unique vectors $\mathbf{u}, \mathbf{v}$ with $|\mathbf{u}|=1$ and $\mathbf{u} \cdot \mathbf{v}=0$ such that $\ell(t)=\mathbf{v}+t \mathbf{u}$. Regarding $\mathbf{u}$ as a point on $S^{2}$ and $\mathbf{v}$ a vector in its tangent space we obtain the isomorphism. There is a natural real structure on the space of oriented real lines namely $(\mathbf{u}, \mathbf{v}) \mapsto(-\mathbf{u}, \mathbf{v})$ which gives rise to the antipodal map on $\mathbb{C} P^{1}$ inside $H^{2}$ as a zero section. Consequently we also obtain a map from the space of holomorphic sections into that of anti-holomorphic sections

$$
r: H^{0}\left(\mathbb{C} P^{1} ; \mathscr{O}(2)\right) \longrightarrow \overline{H^{0}}\left(\mathbb{C} P^{1} ; \mathscr{O}(2)\right) .
$$


A section is called real if it is invariant under this map.

If $z \in H^{2}$ then there is a unique oriented affine line $\ell_{z} \subset \mathbb{R}^{3}$ corresponding to it with a distinguished point $\ell_{z}(0) \in \mathbb{R}^{3}$ (depending on the choice of origin in $\mathbb{R}^{3}$ ). This point can be characterized by the set of all lines in $\mathbb{R}^{3}$ intersecting it which provides us with a real section in $H^{0}\left(\mathbb{C} P^{1} ; \mathscr{O}(2)\right) \cong \mathbb{C}^{3}$ hence is of the form $z([a: b])=\alpha a^{2}+2 \beta a b-\bar{\alpha} b^{2}$ with $\alpha \in \mathbb{C}$ and $\beta \in \mathbb{R}$. Therefore we have two ways of looking at the same object: we can regard $z$ either as a point on the total space $z \in H^{2}$ or as a real section $\alpha a^{2}+2 \beta a b-\bar{\alpha} b^{2} \in H^{0}\left(\mathbb{C} P^{1} ; \mathscr{O}(2)\right)$. Of course, quite tautologically speaking, the section $z$ passes through the point $z$ corresponding to it.

Regarding $z$ as a real section $z([a: b])=\alpha a^{2}+2 \beta a b-\bar{\alpha} b^{2}$ now, this homogeneous polynomial is canonically indentified with the single-valued polynomial in the affine coordinate $\frac{a}{b}$ over $U_{b}$ :

$$
\left.z\right|_{U_{b}}\left(\frac{a}{b}\right)=\alpha\left(\frac{a}{b}\right)^{2}+2 \beta\left(\frac{a}{b}\right)-\bar{\alpha}
$$

Now we define a nowhere vanishing section $\eta^{c}$ of $L^{c}$ by

$$
\eta^{c}(z,[a: b]):= \begin{cases}\mathrm{e}^{c\left(\alpha\left(\frac{a}{b}\right)+\beta\right)} & \text { on } U_{b}^{\prime} ; \\ g(z,[a: b]) \mathrm{e}^{c\left(\alpha\left(\frac{a}{b}\right)+\beta\right)} & \text { on } U_{a}^{\prime} .\end{cases}
$$

It is well-defined since it looks like $\exp c\left(\alpha\left(\frac{a}{b}\right)+\beta\right)$ on $U_{b}^{\prime}$ i.e., where $b \neq 0$ and looks like $\exp c\left(\bar{\alpha}\left(\frac{b}{a}\right)-\beta\right)$ on $U_{a}^{\prime}$ i.e., where $a \neq 0$.

Next we claim that the pair $\left(L^{c}, \eta^{c}\right)$ can be extended uniquely to a pair $\left(\bar{L}^{c}, \bar{\eta}^{c}\right)$ where $\bar{L}^{c}$ is a line bundle over $\bar{\pi}_{z}: P\left(H^{2} \oplus H^{0}\right) \rightarrow \mathbb{C} P^{1}$ which restricts to $L^{c}$ over the finite part as before moreover

$$
\bar{\eta}^{c} \in H^{0}\left(P\left(H^{2} \oplus H^{0}\right) ; \mathscr{E}_{P\left(H^{2} \oplus H^{0}\right)}\left(\bar{L}^{c}\right)\right)
$$

is a section of it which also gives back $\eta^{c}$ on $L^{c}$.

In order to construct these extensions we proceed as follows. First, notice that there is a covering of $P\left(H^{2} \oplus H^{0}\right)$ by four affine charts: the open sets $U_{b}^{\prime}$ and $U_{a}^{\prime}$ of the total space of $H^{2}$ defined above and additional charts $V_{b}^{\prime}$ and $V_{a}^{\prime}$ covering the section at infinity $(w=0)$. Explicitly, let $(z, a)$ and $\left(z^{\prime}, b\right)$ be standard coordinates on $U_{b}^{\prime}$ and $U_{a}^{\prime}$ respectively with $b=a^{-1}$ and $z^{\prime}=z a^{-2}$, then coordinates on $V_{b}^{\prime}$ and $V_{a}^{\prime}$ can be chosen to be $(w, a)$ and $(w, b)$ with $w=z^{-1}$. Since we want to extend the bundle $L^{c}$, the transition function of $\bar{L}^{c}$ from the chart $U_{b}^{\prime}$ to $U_{a}^{\prime}$ must be that of $L^{c}$, i.e. equal to $g=\exp (-c z / a)$. On the other hand, the requirement that $\eta^{c}$ should extend to a section of $\bar{L}^{c}$ fixes the transition function between the charts $U_{b}^{\prime}$ and $V_{b}^{\prime}$ to be $\exp (-c \alpha a-c \beta)$. Similarly, the transition between $U_{a}^{\prime}$ and $V_{a}^{\prime}$ is constrained to be $\exp (c \bar{\alpha} b+c \beta)$. From the cocycle-relation we then deduce that the restriction to the total space of $H^{2}$ (i.e. the open part of $P\left(H^{2} \oplus H^{0}\right)$ away from the section at infinity) of the transition between $V_{b}^{\prime}$ and $V_{a}^{\prime}$ must be identically 1 . In particular, this transition function extends to infinity and the set of transition functions just described yields the line bundle $\bar{L}^{c}$ with a section $\bar{\eta}^{c}$ we were looking for.

Then referring to the notations of Lemma 5.2 (and knowing already that $k=l=s$ and $m=2$ as before) consider the fibration

$$
\bar{\pi}_{x, y}: W \longrightarrow P\left(H^{2} \oplus H^{0}\right)
$$

and use it to pull back both $\bar{L}^{c}$ and $\bar{\eta}^{c}$ over $W$. As an extension of the construction in Lemma 5.2 set

$$
L^{a_{0}, a_{1}, a_{2}, a_{3}, c}:=L^{a_{0}, a_{1}, a_{2}, a_{3}} \otimes \bar{\pi}_{x, y}^{*} \bar{L}^{c}
$$


with $a_{i} \in \mathbb{Z}$ and $c \in \mathbb{C}$; we will also use the convenient notation for the corresponding sheaf:

$$
\mathscr{E}_{W}\left(a_{0}, a_{1}, a_{2}, a_{3}, c\right):=\mathscr{O}_{W}\left(a_{0}, a_{1}, a_{2}, a_{3}\right) \otimes \mathscr{E}_{W}\left(\bar{\pi}_{x, y}^{*} \bar{L}^{c}\right) .
$$

If - again in analogy with Lemma 5.2 - we denote $\bar{\pi}_{x, y}^{*} \bar{\eta}^{ \pm c} \in \mathscr{E}_{W}\left(\bar{\pi}_{x, y}^{*} \bar{L}^{ \pm c}\right)$ simply as $\bar{\eta}^{ \pm c}$ then we have $x \cdot \bar{\eta}^{c} \in \mathscr{E}_{W}(k, 1,0,0, c)$ and $y \cdot \bar{\eta}^{-c} \in \mathscr{E}_{W}(k, 1,0,0,-c)$ etc., and can regard them as twisted coordinates on $W$. Making use of these new twisted coordinates in Lemma 5.2 we define the true compactified singular twistor space $\bar{Z}^{*}$ of the multi-Taub-NUT geometry by the equation $\bar{P}_{c}=0$ where $\bar{P}_{c}: W \rightarrow L^{2 s, 1,1, s, 0}$ looks like

$$
\bar{P}_{c}(x, u, y, v, z, w):=\left(x \cdot \bar{\eta}^{c}\right)\left(y \cdot \bar{\eta}^{-c}\right) w^{s}-\left(u \cdot \bar{\eta}^{c}\right)\left(v \cdot \bar{\eta}^{-c}\right) \prod_{i=1}^{s}\left(z-w p_{i}\right)
$$

which simply gives back (19). Therefore we come up with a singular holomorphic fibration

$$
\bar{\pi}: \bar{Z}^{*} \longrightarrow \mathbb{C} P^{1}
$$

Carrying out desingularization of $\bar{Z}^{*}$ as before we obtain finally that there is a smooth complex fibration

$$
\bar{\pi}: \bar{Z} \longrightarrow \mathbb{C} P^{1}
$$

whose fibers $\bar{X}_{[a: b]}$ are biholomorphic to the rational surfaces constructed in Lemma 5.1. This is the true compact non-singular twistor space of $\left(M_{V}, g_{V}\right)$.

For clarity we remark that from now on, the notations $\bar{Z}^{*}, \bar{Z}, Z^{*}$ and $Z$ will denote the corresponding true twistor spaces of the multi-Taub-NUT geometry (and we will call them simply as the twistor space of the corresponding kind).

Now we are in a position to read off certain cohomology groups of the compactified twistor space.

Lemma 5.4. The middle sheaf cohomology groups of the compactified smooth twistor space $\bar{Z}$ constructed in Lemma 5.3 satisfy

$$
H^{1}\left(\bar{Z} ; \mathscr{O}_{\bar{Z}}(-2)\right) \cong \mathbb{C} \quad \text { and } \quad H^{2}\left(\bar{Z} ; \mathscr{O}_{\bar{Z}}(-2)\right) \cong 0
$$

Proof. First note that in light of Lemma 5.1 all the fibers $\bar{X}_{[a: b]}$ with $[a: b] \in \mathbb{C} P^{1}$ of $(26)$ are rational.

Next, notice that $\mathscr{O}_{\bar{Z}}(-2)=\bar{\pi}^{*} \mathscr{O}(-2)$ is trivial along the fibers of (26). Rationality implies in particular that for all $[a: b]$ and $1 \leq q \leq 2$ one has

$$
H^{q}\left(\bar{X}_{[a: b]} ;\left(\left.\bar{\pi}\right|_{\bar{X}_{[a: b]}}\right)^{*} \mathscr{O}(-2)\right) \cong H^{q}\left(\bar{X}_{[a: b]} ; \mathscr{O}_{\bar{X}_{[a: b]}}\right)=0
$$

so $R^{q} \bar{\pi}_{*} \bar{\pi}^{*} \mathscr{O}(-2)$ is the zero sheaf. On the other hand $R^{0} \bar{\pi}_{*} \bar{\pi}^{*} \mathscr{O}(-2) \cong \mathscr{O}(-2)$ by the projection formula consequently

$$
H^{0}\left(\mathbb{C} P^{1} ; R^{0} \bar{\pi}_{*} \bar{\pi}^{*} \mathscr{O}(-2)\right)=0 \quad \text { and } \quad H^{1}\left(\mathbb{C} P^{1} ; R^{0} \bar{\pi}_{*} \bar{\pi}^{*} \mathscr{O}(-2)\right)=\mathbb{C}
$$


The second level of the Leray spectral sequence associated to $(26)$ and the sheaf $\pi^{*} \mathscr{O}(-2)$ is given by $E_{2}^{p, q}=H^{p}\left(\mathbb{C} P^{1} ; R^{q} \bar{\pi}_{*} \bar{\pi}^{*} \mathscr{O}(-2)\right)$. Taking into account our calculations so far, the only non-trivial part is a one-dimensional space in bidegree $(1,0)$ :

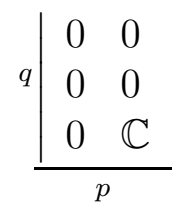

implying that the spectral sequence collapses at this step and the statement follows. $\diamond$

Keeping these results in mind now we recall the notion of the "global Ext" groups $\operatorname{Ext}^{k}(V ; \mathscr{R}, \mathscr{S})$ over a complex manifold $V$ with two coherent sheaves $\mathscr{R}$ and $\mathscr{S}$ on it. The background material can be found in [16]. These objects are appropriate generalizations of the sheaf cohomology groups over complex manifolds. For instance if $\mathscr{R}=\mathscr{O}_{V}$ then we know that

$$
\operatorname{Ext}^{k}\left(V ; \mathscr{O}_{V}, \mathscr{S}\right) \cong H^{k}(V ; \mathscr{S}) .
$$

If $V$ is compact $n$ dimensional then there is a generalization of the Serre duality theorem for coherent sheaves, called the Grothendieck duality theorem:

$$
H^{k}(V ; \mathscr{S}) \cong\left(\operatorname{Ext}^{n-k}\left(V ; \mathscr{S}, \mathscr{K}_{V}\right)\right)^{*}
$$

and in the same fashion if $\mathscr{S}=\mathscr{O}(E)$ is locally free then the Serre duality theorem implies that

$$
\left(\operatorname{Ext}^{n-k}\left(V ; \mathscr{O}(E), \mathscr{K}_{V}\right)\right)^{*} \cong H^{n-k}\left(V ; \mathscr{O}\left(E^{*}\right) \otimes \mathscr{K}_{V}\right)
$$

We will be particularly interested in $\operatorname{Ext}^{1}(V ; \mathscr{R}, \mathscr{S})$ which classifies extensions of coherent sheaves over $V$ :

$$
0 \longrightarrow \mathscr{S} \longrightarrow \mathscr{E} \longrightarrow \mathscr{R} \longrightarrow 0
$$

Following Atiyah [2] we obtain:

Lemma 5.5. Let $\mathscr{J}_{Y}$ denote the ideal sheaf of holomorphic functions on $\bar{Z}$ vanishing on a fixed real line $Y \subset Z \subset \bar{Z}$. Then

$$
\operatorname{Ext}^{1}\left(\bar{Z} ; \mathscr{J}_{Y}, \mathscr{O}_{\bar{Z}}(-2)\right) \cong \mathbb{C}^{2}
$$

Proof. Note that $\mathscr{O}_{Y}(-2) \cong \mathscr{K}_{Y}$, the canonical sheaf of $Y$. Let $j: Y \rightarrow \bar{Z}$ be the inclusion and denote by $j_{*} \mathscr{K}_{Y}$ the extension of $\mathscr{K}_{Y}$ by zero over $\bar{Z}$. Hence $j_{*} \mathscr{K}_{Y}$ is a coherent sheaf on $\bar{Z}$. Applying (28) on $\bar{Z}$ in the last step we obtain

$$
H^{k}\left(Y ; \mathscr{K}_{Y}\right) \cong H^{k}\left(\bar{Z} ; j_{*} \mathscr{K}_{Y}\right) \cong\left(\operatorname{Ext}^{3-k}\left(\bar{Z} ; j_{*} \mathscr{K}_{Y}, \mathscr{K}_{\bar{Z}}\right)\right)^{*}
$$

and additionally we know that

$$
\operatorname{Ext}^{3-k}\left(\bar{Z} ; j_{*} \mathscr{K}_{Y}, \mathscr{K}_{\bar{Z}}\right) \cong \operatorname{Ext}^{3-k}\left(\bar{Z} ; j_{*} \mathscr{O}_{Y} \otimes \bar{\pi}^{*} \mathscr{K}_{Y}, \mathscr{K}_{\bar{Z}}\right) \cong \operatorname{Ext}^{3-k}\left(\bar{Z} ; j_{*} \mathscr{O}_{Y}, \bar{\pi}^{*} \mathscr{K}_{Y}^{*} \otimes \mathscr{K}_{\bar{Z}}\right)
$$

We already know that $\left.\left(\bar{\pi}^{*} K_{Y}^{*} \otimes K_{\bar{Z}}\right)\right|_{Y}$ is non-canonically isomorphic to $H^{-2}$, therefore $\mathscr{O}_{\bar{Z}}(-2)$ is an extension of $\left.\left(\bar{\pi}^{*} \mathscr{K}_{Y}^{*} \otimes \mathscr{K}_{\bar{Z}}\right)\right|_{Y}$ over the whole $\bar{Z}$ that is,

$$
\operatorname{Ext}^{3-k}\left(\bar{Z} ; j_{*} \mathscr{O}_{Y}, \bar{\pi}^{*} \mathscr{K}_{Y}^{*} \otimes \mathscr{K}_{\bar{Z}}\right) \cong \operatorname{Ext}^{3-k}\left(\bar{Z} ; j_{*} \mathscr{O}_{Y}, \mathscr{O}_{\bar{Z}}(-2)\right)
$$


Therefore, taking into account that $H^{2}\left(Y ; \mathscr{K}_{Y}\right) \cong 0$ and $H^{1}\left(Y ; \mathscr{K}_{Y}\right) \cong \mathbb{C}$ we conclude that

$$
\operatorname{Ext}^{1}\left(\bar{Z} ; j_{*} \mathscr{O}_{Y}, \mathscr{O}_{\bar{Z}}(-2)\right) \cong 0 \text { and } \operatorname{Ext}^{2}\left(\bar{Z} ; j_{*} \mathscr{O}_{Y}, \mathscr{O}_{\bar{Z}}(-2)\right) \cong \mathbb{C}
$$

Referring to $(27)$ we know $\operatorname{Ext}^{i}\left(\bar{Z} ; \mathscr{O}_{\bar{Z}}, \mathscr{O}_{\bar{Z}}(-2)\right) \cong H^{i}\left(\bar{Z} ; \mathscr{O}_{\bar{Z}}(-2)\right)$ consequently Lemma 5.4 additionally yields

$$
\operatorname{Ext}^{1}\left(\bar{Z} ; \mathscr{O}_{\bar{Z}}, \mathscr{O}_{\bar{Z}}(-2)\right) \cong \mathbb{C} \quad \text { and } \quad \operatorname{Ext}^{2}\left(\bar{Z} ; \mathscr{O}_{\bar{Z}}, \mathscr{O}_{\bar{Z}}(-2)\right) \cong 0
$$

Now consider the short exact sequence of coherent sheaves over $\bar{Z}$ :

$$
0 \longrightarrow \mathscr{J}_{Y} \longrightarrow \mathscr{O}_{\bar{Z}} \longrightarrow j_{*} \mathscr{O}_{Y} \longrightarrow 0
$$

A segment of the associated long exact sequence of the global Ext groups with $\mathscr{O}_{\bar{Z}}(-2)$ looks like

$$
\begin{aligned}
\ldots & \longrightarrow \operatorname{Ext}^{1}\left(\bar{Z} ; j_{*} \mathscr{O}_{Y}, \mathscr{O}_{\bar{Z}}(-2)\right) \longrightarrow \operatorname{Ext}^{1}\left(\bar{Z} ; \mathscr{O}_{\bar{Z}}, \mathscr{O}_{\bar{Z}}(-2)\right) \longrightarrow \\
& \longrightarrow \operatorname{Ext}^{1}\left(\bar{Z} ; \mathscr{J}_{Y}, \mathscr{O}_{\bar{Z}}(-2)\right) \quad \longrightarrow \quad \operatorname{Ext}^{2}\left(\bar{Z} ; j_{*} \mathscr{O}_{Y}, \mathscr{O}_{\bar{Z}}(-2)\right) \longrightarrow \\
& \longrightarrow \operatorname{Ext}^{2}\left(\bar{Z} ; \mathscr{O}_{\bar{Z}}, \mathscr{O}_{\bar{Z}}(-2)\right) \longrightarrow
\end{aligned}
$$

which gives

$$
0 \longrightarrow \mathbb{C} \longrightarrow \operatorname{Ext}^{1}\left(\bar{Z} ; \mathscr{J}_{Y}, \mathscr{O}_{\bar{Z}}(-2)\right) \longrightarrow \mathbb{C} \longrightarrow 0
$$

providing the result. $\diamond$

After these algebro-geometric preliminaries the time has come to relate our considerations so far with anti-instantons over a multi-Taub-NUT space.

First we establish a link with harmonic functions. The embedding $i: Z \backslash Y \subset \bar{Z}$ together with (27) implies a homomorphism

$$
i^{*}: \operatorname{Ext}^{1}\left(\bar{Z} ; \mathscr{J}_{Y}, \mathscr{O}_{\bar{Z}}(-2)\right) \longrightarrow \operatorname{Ext}^{1}\left(Z \backslash Y ; \mathscr{J}_{Y}, \mathscr{O}_{Z \backslash Y}(-2)\right) \cong H^{1}\left(Z \backslash Y ; \mathscr{O}_{Z \backslash Y}(-2)\right) .
$$

However for a fixed real line $Y \subset Z$ representing the point $y \in M_{V}$ via Penrose transform we have an isomorphism

$$
T:\left.H^{1}\left(Z \backslash Y ; \mathscr{O}_{Z \backslash Y}(-2)\right) \cong \operatorname{Ker} \triangle_{g_{V}}\right|_{M_{V} \backslash\{y\}}
$$

Consequently one can think of the elements of $\operatorname{Ext}^{1}\left(\bar{Z} ; \mathscr{J}_{Y}, \mathscr{O}_{\bar{Z}}(-2)\right)$ as harmonic functions. Therefore Lemma 5.5 provides us with a distinguished 2-parameter family of complex-valued harmonic functions on $M_{V} \backslash\{y\}$. These functions can easily be found by observing that the positive minimal Green function with singularity in $y \in M_{V}$ of Defintion 4.1 provides us with a splitting of $\operatorname{Ext}^{1}\left(\bar{Z} ; \mathscr{J}_{Y}, \mathscr{O}_{\bar{Z}}(-2)\right) \cong \mathbb{C}^{2}$ as a vector space into two one dimensional summands. More precisely (30) gives

$$
0 \longrightarrow \operatorname{Ext}^{1}\left(\bar{Z} ; \mathscr{O}_{\bar{Z}}, \mathscr{O}_{\bar{Z}}(-2)\right) \longrightarrow \operatorname{Ext}^{1}\left(\bar{Z} ; \mathscr{J}_{Y}, \mathscr{O}_{\bar{Z}}(-2)\right) \stackrel{\delta}{\longrightarrow} \operatorname{Ext}^{2}\left(\bar{Z} ; j_{*} \mathscr{O}_{Y}, \mathscr{O}_{\bar{Z}}(-2)\right) \longrightarrow 0
$$

and by $[2$, Theorem 1] the Green function defines a map

$$
\operatorname{Ext}^{2}\left(\bar{Z} ; j_{*} \mathscr{O}_{Y}, \mathscr{O}_{\bar{Z}}(-2)\right) \longrightarrow \operatorname{Ext}^{1}\left(\bar{Z} ; \mathscr{J}_{Y}, \mathscr{O}_{\bar{Z}}(-2)\right)
$$

so that all elements of the middle term canonically can be written in the form $\alpha+\beta$ with the first factor $\alpha$ coming from $\operatorname{Ext}^{1}\left(\bar{Z} ; \mathscr{O}_{\bar{Z}}, \mathscr{O}_{\bar{Z}}(-2)\right)$ which is independent of $Y$ hence corresponds to a constant harmonic function $\mu \in \mathbb{C}$ on $\left(M_{V}, g_{V}\right)$ while $\beta=\lambda G(\cdot, y)$ with $\lambda \in \mathbb{C}$.

Consequently we obtain the following (also cf. [2]): 
Theorem 5.1. Fix a point $y \in M_{V}$ of the multi-Taub-NUT space $\left(M_{V}, g_{V}\right)$ and consider the associated real line $Y \subset Z$ and the group $\operatorname{Ext}^{1}\left(\bar{Z} ; \mathscr{J}_{Y}, \mathscr{O}_{\bar{Z}}(-2)\right) \cong \mathbb{C}^{2}$. Then any element of this group can canonically be written in the form $\mu+\lambda G(\cdot, y)$ where $G(\cdot, y)$ is the unique minimal positive Green function concentrated at $y \in M_{V}$ and $\mu, \lambda \in \mathbb{C}$ are constants.

Remark. Notice that in fact the above theorem holds for any anti-half-conformally flat nonparabolic manifold whose twistor space admits a compactification satisfying Lemma 5.4.

One can then see that the positive harmonic functions of (9) - from which our anti-instantons $\left[\nabla_{\tilde{A}_{y, \lambda}}\right]$ stem - can also be regarded as elements of $\operatorname{Ext}^{1}\left(\bar{Z} ; \mathscr{J}_{Y}, \mathscr{O}_{\bar{Z}}(-2)\right)$ hence they describe extensions of two coherent sheaves

$$
0 \longrightarrow \mathscr{O}_{\bar{Z}}(-2) \longrightarrow \overline{\mathscr{F}}(-1) \longrightarrow \mathscr{J}_{Y} \longrightarrow 0 \text {. }
$$

Restricting this to $Z$, the resulting sheaves $\mathscr{F}(-1)$ will be locally free hence provide us with rank 2 holomorphic vector bundles $F(-1)$ over $Z$.

Our aim is now to understand those twisted vector bundles together with their canonical sections among the aforementioned vector bundles over $Z$ which are associated to pure Green functions. We want to show that for two points $y, \mathrm{e}^{\mathbf{i} \tau} y \in M_{V}$ on the same $S^{1}$-orbit, these twisted vector bundles are isomorphic. For this purpose we have to construct the real structure on $Z$ which gives rise to the multi-Taub-NUT geometry. It is sufficient to work over the open twistor space $Z \subset \bar{Z}$, because we are only interested in the real lines lying over points $y \in M_{V}$ at finite distance. Therefore we take a closer look at this space.

Restricting our construction in Lemma 5.3 and in particular the singular fibration (25) to the finite part we obtain a model for the non-compact singular twistor space $\pi: Z^{*} \rightarrow \mathbb{C} P^{1}$ as the hypersurface $P_{c}=0$ where $P_{c}:\left(H^{s} \otimes L^{c}\right) \oplus\left(H^{s} \otimes L^{-c}\right) \oplus H^{2} \rightarrow H^{2 s}$ takes the shape

$$
P_{c}(x, y, z):=\left(x \cdot \eta^{c}\right)\left(y \cdot \eta^{-c}\right)-\prod_{j=1}^{s}\left(z-p_{j}\right) .
$$

(For clarity we remark that in (31) and from now on without introducing extra notation all the bundles $H^{k}$ over $\mathbb{C} P^{1}$ will be pulled back to $H^{2}$.)

But first we make a digression on the choice $k=l=s$ and $m=2$ in Lemmata 5.2 and 5.3 and note that it is also dictated by the requirement that the normal bundle of a real line $Y \subset Z$ must be isomorphic to $H \oplus H$ and $Z$ must possess a non-trivial real structure leading to the multi-Taub-NUT geometry.

Indeed, to describe the normal bundle of a real line $Y \subset Z$ is equivalent to describe that of a generic real line $Y \subset Z^{*}$. Since it is a section of the bundle $\pi: Z^{*} \rightarrow \mathbb{C} P^{1}$ its normal bundle is the restriction of the tangent bundle of $H^{s} \oplus H^{s} \oplus H^{2}$ to a generic (non-singular) line i.e., the kernel of the map $\left(P_{x}, P_{y}, P_{z}\right): T\left(H^{s} \oplus H^{s} \oplus H^{2}\right) \rightarrow T H^{2 s}$. This shows that $\left\langle c_{1}\left(N_{Y}\right),[Y]\right\rangle=s+s+2-2 s=2$ moreover the type of the normal bundle is stable under small perturbations yielding $N_{Y} \cong H \oplus H$. An important consequence is that since $\left.K_{Z}\right|_{Y} \cong K_{Y} \otimes \Lambda^{2} N_{Y}^{*}$ we therefore find $K_{Z} \cong \pi^{*} H^{-4}$ for the canonical bundle of $Z$ as we already noted in Sect. 2 . Notice that this relation does not extend to the compactification $\bar{Z}$. Indeed, for every real line $Y$ at finite distance the relation $N_{Y} \cong H \oplus H$ continues to hold, but because the fibers of the map (26) become compact, in $K_{\bar{Z}}$ there will be a twist coming from the canonical bundle of $\bar{X}$ as well. This explains the asymmetry between the middle cohomology groups in Lemma 5.4.

Next we move to the construction of the real structure on $Z$ following [10] — originally due to $[6,22,24]$ - which gives rise to the multi-Taub-NUT geometry. As we have seen in Sect. 2 the 
real structure on our twistor space must be induced by the antipodal map on $\mathbb{C} P^{1}$. Therefore, assuming $c \in \mathbb{R}$, we use (24) to construct induced maps

$$
r_{c}: H^{0}\left(H^{2} ; \mathscr{E}_{H^{2}}\left(H^{s} \otimes L^{c}\right)\right) \longrightarrow H^{0}\left(H^{2} ; \mathscr{E}_{H^{2}}\left(H^{s} \otimes L^{-c}\right)\right)
$$

satisfying $r_{c}^{2}=(-1)^{s} \mathrm{Id}$. The real structure $\tau_{c}: Z^{*} \rightarrow Z^{*}$ on the twistor space given by $P_{c}=0$ in (31) is defined to be

$$
\tau_{c}(x, y, z):=\left((-1)^{s} r_{c}\left(y \cdot \eta^{-c}\right), r_{c}\left(x \cdot \eta^{c}\right),-r(z)\right) .
$$

A twistor line is called real if it is invariant under this map. We claim that the space $Z^{*}$ constructed in Lemma 5.3 together with this $\tau_{c}$ is indeed (a singular model of) the twistor space of the multi-Taub-NUT geometry. The simplest way of demonstrating this would be the derivation of the multi-Taub-NUT metric (6) via Penrose' non-linear graviton construction outlined in Sect. 2. However, after a considerable hesitation we decided not to present this calculation here because it is quite long moreover it is already available in the literature for a long time ([6, pp. 393-395], [22, 24]).

We rather find the corresponding real lines by a factorization method ([6, pp. 393-395] and [22]). Using the notations and constructions of Lemma 5.3 if we encode the NUT $q_{j} \in \mathbb{R}^{3}$ as the real section of $H^{2}$ whose shape over $U_{b} \subset \mathbb{C} P^{1}$ looks like $\left.p_{j}\left(\left[\frac{a}{b}: 1\right]\right)\right|_{U_{b}}=\alpha_{j}\left(\frac{a}{b}\right)^{2}+2 \beta_{j}\left(\frac{a}{b}\right)-\bar{\alpha}_{j}$ as well as write a general point in $\mathbb{R}^{3}$ as $\left.\zeta\left(\left[\frac{a}{b}: 1\right]\right)\right|_{U_{b}}=\alpha\left(\frac{a}{b}\right)^{2}+2 \beta\left(\frac{a}{b}\right)-\bar{\alpha}$ then the roots $\rho_{j}, \sigma_{j} \in \mathbb{C} \subset \mathbb{C} P^{1}$ of the equation $\zeta-p_{j}=0$ are

$$
\rho_{j}:=\frac{-\left(\beta-\beta_{j}\right)-\sqrt{\left(\beta-\beta_{j}\right)^{2}+\left|\alpha-\alpha_{j}\right|^{2}}}{\alpha-\alpha_{j}}, \quad \sigma_{j}:=\frac{-\left(\beta-\beta_{j}\right)+\sqrt{\left(\beta-\beta_{j}\right)^{2}+\left|\alpha-\alpha_{j}\right|^{2}}}{\alpha-\alpha_{j}} .
$$

The real lines appear by simply factorizing in a $\tau_{c}$-invariant way the equation (31) and imposing the reality condition (this already has been done for $\zeta$ ). They are of the form

$$
\left\{\begin{array}{l}
\xi(\zeta,[a: b])=A \eta^{c}(\zeta,[a: b]) \prod_{j=1}^{s}\left(a-\rho_{j} b\right) \\
v(\zeta,[a: b])=B \eta^{-c}(\zeta,[a: b]) \prod_{j=1}^{s}\left(a-\sigma_{j} b\right) \\
\zeta([a: b])=\alpha a^{2}+2 \beta a b-\bar{\alpha} b^{2} .
\end{array}\right.
$$

To see that this is indeed a $\tau_{c}$-invariant factorization, the only non-trivial fact we have to check is that $\eta^{-c}$ goes to $\overline{\eta^{c}}$ if $c \in \mathbb{R}$ and vice versa on both charts $U_{a}^{\prime}$ and $U_{b}^{\prime}$. However for example for $[a: b] \in U_{a}^{\prime}$ we have $[-\bar{b}: \bar{a}] \in U_{b}^{\prime}$, and $\eta^{-c}(\zeta,[-\bar{b}: \bar{a}])=\exp \left(\overline{c\left(\bar{\alpha}\left(\frac{b}{a}\right)-\beta\right)}\right)$ which is just $\overline{\eta^{c}(\zeta,[a: b])}$ written on the chart $U_{a}^{\prime}$. The coefficients $A, B$ here are arbitrary constants satisfying $A B=\prod\left(\alpha-\alpha_{i}\right)$, the leading coefficient of $\prod\left(\zeta-p_{i}\right)$. As this latter number is non-zero for $\alpha \neq \alpha_{j}$, say $B$ can be expressed in terms of $A$ and $\alpha$. But the reality constraint moreover implies

$$
|A|^{2}=\prod_{j=1}^{s}\left(\left(\beta-\beta_{j}\right)+\sqrt{\left(\beta-\beta_{j}\right)^{2}+\left|\alpha-\alpha_{j}\right|^{2}}\right) .
$$

Hence the set of these real lines is locally parameterized ${ }^{4}$ by $\arg A \in S^{1}$ and $(\operatorname{Re} \alpha, \operatorname{Im} \alpha, \beta) \in \mathbb{R}^{3}$ and provides us with a local chart of $M_{V}$ as it was constructed in Sect. 3. The choice $c=0$

\footnotetext{
${ }^{4}$ Note that the way of assigning the roots of $\zeta-p_{j}$ to $\xi$ and $v$ is well-defined only up to the action of the Galois group of the problem which explains that we have a local parameterization only.
} 
gives rise to the ALE $A_{s-1}$ geometries while $c>0$ real provides us with the ALF $A_{s-1}$ i.e., the multi-Taub-NUT spaces. Hence from now on we will restrict to $c=1$ in accord with previous sections. A given real line will be denoted as $Y_{\alpha, \beta, A}$.

The next step is to express the transition matrices for the vector bundle $F_{\alpha, \beta, A}(-1)$ on $Z$ as well as its section $s_{\alpha, \beta, A} \in H^{0}\left(Z ; \mathscr{O}_{Z}\left(F_{\alpha, \beta, A}(-1)\right)\right)$, associated to the canonical element

$$
G\left(\cdot, y_{\alpha, \beta, A}\right) \in \operatorname{Ext}^{1}\left(Z \backslash Y_{\alpha, \beta, A} ; \mathscr{J}_{Y_{\alpha, \beta, A}}, \mathscr{O}_{Z \backslash Y_{\alpha, \beta, A}}(-2)\right)
$$

representing the Green function. Here, we follow again [2] and first work over $Z^{*}$. Set

$$
\begin{aligned}
& f:=x \cdot \eta^{1}-\xi \\
& g:=y \cdot \eta^{-1}-v \\
& h:=z-\zeta .
\end{aligned}
$$

By definition, the real twistor line $Y_{\alpha, \beta, A}$ is given by the equations

$$
f=g=h=0 .
$$

Define open subsets $U_{\xi}$ and $U_{v}$ by $\xi \neq 0$ and $v \neq 0$ respectively. Then, restricted to $U_{\xi} \cap Z^{*}$ the real line is given by the complete intersection $f=h=0$ as well as restricted to $U_{v} \cap Z^{*}$ it is the complete intersection $g=h=0$. Furthermore, for all generic $\zeta$ the polynomials $\zeta-p_{i}$ and $\zeta-p_{j}$ do not have common roots for $i \neq j$, so $U_{\xi}$ and $U_{v}$ cover $Z^{*}$. We restrict to such choices $\alpha, \beta, A$, but by continuity our results continue to hold in the general case too. Let us now set

$$
\theta:=\frac{\left(x \cdot \eta^{1}\right)\left(y \cdot \eta^{-1}\right)-\xi v}{h}=\frac{x y-\xi v}{h},
$$

a polynomial in $a, b$. Straightforward verification yields the identities

$$
f=-\frac{x \cdot \eta^{1}}{v} g+\frac{\theta}{v} h \quad \text { and } \quad h=-\frac{h}{v} g+\frac{y \cdot \eta^{-1}}{v} h .
$$

Obviously $f, g$ and $h$ are well-defined sections, vanishing on $Y_{\alpha, \beta, A}$, of $H^{s} \otimes L^{1}, H^{s} \otimes L^{-1}$ and $H^{2}$ respectively.

Hence, we can define a rank 2 vector bundle $F_{\alpha, \beta, A}(-1)$ on $Z^{*}$ by gluing the sections $f$ and $h$ of the bundle $\left(H^{s} \otimes L^{1}\right) \oplus H^{2}$ restricted to $U_{\xi}$ with the sections $g$ and $h$ of the bundle $\left(H^{s} \otimes L^{-1}\right) \oplus H^{2}$ on $U_{v}$ using the gluing matrix

$$
M_{\alpha, \beta, A}:=\frac{1}{v}\left(\begin{array}{cc}
-x \cdot \eta^{1} & \theta \\
-h & y \cdot \eta^{-1}
\end{array}\right)
$$

on $U_{\xi} \cap U_{v}$. Moreover, we get a section $s_{\alpha, \beta, A}$ of this vector bundle by setting it to be equal to $(f, h)$ on $U_{\xi}$ and to $(g, h)$ on $U_{v}$. This section vanishes precisely on $Y_{\alpha, \beta, A}$. This bundle provides us with a Green function hence is positive [17, 21,34] moreover it is the minimal positive one since from [2, Eq. 7.11] one can check that it converges to zero at infinity.

Recall also that $S^{1}$ acts on $M_{V}$ as identified with the space of real sections by multiplication on the component $A$, or equivalently in view of the identity $A B=\prod\left(\alpha-\alpha_{i}\right)$, by inverse multiplication on $B$. Our aim is to show that if we consider the twistor line associated to a point $\left(\alpha, \beta, \mathrm{e}^{\mathrm{i} \tau} A\right)$, then the vector bundle $F(-1)$ constructed for this point will be isomorphic to the 
one corresponding to $(\alpha, \beta, A)$. But the gluing matrix (33) for these former data is related to that of the latter ones via

$$
M_{\alpha, \beta, \mathrm{e}^{\mathbf{i} \tau} A}=\mathrm{e}^{-\mathbf{i} \tau} M_{\alpha, \beta, A} .
$$

It follows that the change of trivializations $(f, h) \mapsto\left(\mathrm{e}^{\mathbf{i} \tau} f, \mathrm{e}^{\mathbf{i} \tau} h\right)$ on $U_{\xi}$ induces an isomorphism between the bundles $F_{\alpha, \beta, A}(-1)$ and $F_{\alpha, \beta, \mathrm{e}^{\mathrm{i} \tau} A}(-1)$.

As we have seen we obtain $Z$ from the singular space $Z^{*}$ by blowing it up in finitely many points. Carrying out this procedure and then pulling back the bundles just constructed we come up with isomorphic vector bundles $F_{\alpha, \beta, A}(-1)$ and $F_{\alpha, \beta, \mathrm{e}^{\mathbf{i} \tau} A}(-1)$ over the regular (and non-compact) twistor space $Z$.

Consider the untwisted vector bundle $F_{\alpha, \beta, A}=F_{\alpha, \beta, A}(-1) \otimes H$. First of all, it is clear that it is a holomorphic rank 2 vector bundle over $Z$.

Secondly, the restriction of $F_{\alpha, \beta, A}$ to any real line in $Z$ is holomorphically trivial. This follows since the bundle we have constructed just corresponds to the Green function and this function is everywhere positive as we already noted above.

Thirdly, the anti-linear map (32) induces a real structure on $F_{\alpha, \beta, A}(-1)$ over $\tau_{1}$ simply because $\tau_{1}$ switches $H^{s} \otimes L^{1}$ and $H^{s} \otimes L^{-1}$ while maps $H^{2}$ to $H^{2}$. Therefore, since over $\mathbb{C} P^{1}$ the bundle $H$ carries a symplectic structure [18], the untwisted bundle $F_{\alpha, \beta, A}=F_{\alpha, \beta, A}(-1) \otimes H$ also carries a symplectic structure $\tilde{\tau}_{1}: F_{\alpha, \beta, A} \rightarrow F_{\alpha, \beta, A}$ lying over $\tau_{1}$.

Since the same conclusions are also true for $F_{\alpha, \beta, \mathrm{e}^{\mathbf{i} \tau} A}$ it then follows that the corresponding untwisted vector bundles satisfy the three conditions of the Atiyah-Ward correspondence (cf. the summary of the Atiyah-Ward correspondence in Sect. 2) hence they indeed give rise to antiinstantons. Since $F_{\alpha, \beta, A}$ and $F_{\alpha, \beta, \mathrm{e}^{\mathrm{i} \tau} A}$ are isomorphic, we therefore conclude that the limiting anti-instantons are gauge equivalent i.e., $\left[\nabla_{\tilde{A}_{y,+\infty}}\right]=\left[\nabla_{\tilde{A}_{\mathrm{e} i \tau}{ }_{y,+\infty}}\right]$ as desired.

\section{Conclusion}

In this paper we considered $\mathrm{SU}(2)$ anti-instanton moduli spaces over the multi-Taub-NUT spaces containing solutions with integer energy. As a consequence of the general theory [13] in principle anti-instantons with the same nice properties but with fractional energy also may exist over these spaces. The tantalazing question therefore arises whether or not these other moduli spaces are empty.

\section{References}

[1] Anderson, M.T., Kronheimer, P.B., LeBrun, C.: Complete Ricci-flat manifolds with infinite topological type, Comm. Math. Phys. 125, 637-642 (1989);

[2] Atiyah, M.F.: Green's functions for self-dual four-manifolds, Mathematical analysis and applications, Part A, Advances in mathematics supplementary studies 7A, 129-158 (1981);

[3] Atiyah, M.F., Hitchin, N.J.: The geometry and dynamics of magnetic monopoles, Princeton University Press, Princeton (1988);

[4] Atiyah, M.F., Hitchin, N.J., Singer, I.M.: Self-duality in four-dimensional Riemannian geometry, Proc. Roy. Soc. London A362, 425-461 (1978); 
[5] Atiyah. M.F., Ward, R.: Instantons and algebraic geometry, Comm. Math. Phys. 55, 117124 (1977);

[6] Besse, A.L.: Einstein Manifolds, Springer-Verlag, Berlin (1987);

[7] Biquard, O., Jardim, M.: Asymptotic behaviour and moduli space of double periodic instantons, Journ. Eur. Math. Soc. 3, 335-375 (2001);

[8] Cherkis, S.: Moduli spaces of instantons on the Taub-NUT space, preprint, 25 pp., arXiv: hep-th/0805.1245 (2008);

[9] Cherkis, S., Hitchin, N.J.: Gravitational instantons of type $D_{k}$, Comm. Math. Phys. 260, 299-317 (2005);

[10] Etesi, G.: Classification of 't Hooft instantons over multi-centered gravitational instantons, Nucl. Phys. B662, 511-530 (2003);

[11] Etesi, G.: The topology of asymptotically locally flat gravitational instantons, Phys. Lett. B641, 461-465 (2006);

[12] Etesi, G., Hausel, T.: On Yang-Mills-instantons over multi-centered gravitational instantons, Comm. Math. Phys. 235, 275-288 (2003), arXiv: hep-th/0207196;

[13] Etesi, G., Jardim, M.: Moduli spaces of self-dual connections over asymptotically locally flat gravitatinal instantons, Comm. Math. Phys. 280, 285-313 (2008), Erratum: ibid. 288, 799-800 (2009);

[14] Freedman, M.H.: The topology of four-manifolds, Journ. Diff. Geom. 17, 357-454 (1982);

[15] Gibbons, G.W., Hawking, S.W.: Gravitational multi-instantons, Phys. Lett. B78, 430-432 (1978);

[16] Griffiths, Ph., Harris, J.: Principles of Algebraic Geometry, John Wiley \& Sons, Inc., New York (1984);

[17] Grigor'yan, A.A.: The existence of positive fundamental solutions of the Laplace equation on Riemannian manifolds (Russian), Math. Sb. (S.N.) 128 (170), 354-363, 446 (1985);

[18] Hartshorne, R.: Stable vector bundles and instantons, Comm. Math. Phys. 59, 1-15 (1978);

[19] Hausel, T., Hunsicker, E., Mazzeo, R.: Hodge cohomology of gravitational instantons, Duke Math. Journ. 122, 485-548 (2004);

[20] Hawking, S.W.: Gravitational instantons, Phys. Lett. A60, 81-83 (1977);

[21] Hebey, E.: Nonlinear analysis on manifolds: Sobolev spaces and inequalities, Courant Lecture Notes 5, AMS (2000);

[22] Hitchin, N.J.: Polygons and gravitons, Math. Proc. Camb. Phyl. Soc. 85, 465-476 (1979);

[23] Hitchin, N.J.: Linear field equations on self-dual spaces, Proc. Roy. Soc. London A370, 173-191 (1980); 
[24] Hitchin, N.J.: Twistor construction of Einstein metrics, in: Global Riemannian geometry (Durham, 1983), 115-125, Ellis Horwood Ser.: Math. Appl., Horwood, Chichester (1984);

[25] Jackiw, R., Nohl, C., Rebbi, C.: Conformal properties of pseudo-particle configurations, Phys. Rev. D15, 1642-1646 (1977);

[26] Kronheimer, P.B: The construction of ALE spaces as hyper-Kähler quotients, Journ. Diff. Geom. 29 665-683 (1989);

[27] Kronheimer, P.B., Nakajima, H.: Yang-Mills instantons on ALE gravitational instantons, Math. Ann. 288, no.2., 263-607 (1990);

[28] LeBrun, C.: Complete Ricci-flat Kähler metrics on $\mathbb{C}^{n}$ need not be flat, Proc. Symp. Pure Math. 52 (2), 297-304 (1991);

[29] Minerbe, V.: On some asymptotically flat manifolds with non-maximal volume growth, preprint, 49 pp., arXiv: 0709.1084 [math.DG] (2007);

[30] Minerbe, V.: A mass for ALF manifolds, Comm. Math. Phys. 289, 925-955 (2009);

[31] Page, D.N.: Green's function for gravitational multi-instantons, Phys. Lett. B85, 369-372 (1979);

[32] Penrose, R.: Nonlinear gravitons and curved twistor theory, Gen. Rel. Grav. 7, 31-52 (1976);

[33] Uhlenbeck, K.K.: Removable singularities in Yang-Mills fields, Comm. Math. Phys. 83, 11-29 (1982);

[34] Varopoulos, N.: Potential theory and diffusion on Riemannian manifolds, Conference on harmonic analysis in honor of Antoni Zygmund (Chicago, Ill. 1981), Vol. I, II, 821-837, Wadsworth Math. Ser., Wadsworth, Belmont, USA (1983). 